\title{
Medial pivot versus (cam post) posterior stabilised total knee arthroplasty, systematic review and meta-analysis of 3837 knees
}

\author{
Hany Elbardesy, Hazem M Salamah, André McLeod, Pawan Kumar Thada, Eman Reda Mohammed, \\ Fatima Alzhra Hanifa, Mostafa Roshdy, Shane Guerin
}

From the Department of trauma and orthopaedic, Cork University Hospital, Wilton, Cork, Ireland

In the current literature, there is no consensus as to whether the medial pivot (MP) or posterior-stabilised (PS) knee provides the best result for the patient in the context of post-operative range of motion (ROM) and patient reported outcome measures (PROMs). The aim of this systematic review is to provide this equipoise with some clarity. We conducted this study following both the Preferred Reporting Items for Systematic Reviews and Meta-analyses Statement (PRISMA) and the Cochrane Handbook for systematic reviews and meta-analysis. Studies comparing the MP and PS knees from all regions and written in any language were included. Twentyone studies were included in this meta-analysis. They were prepared and analysed using Review Manager V5.0 [Computer Program] (RevMan5). We calculated the risk ratio to measure the treatment effect, taking the heterogeneity of the studies into consideration. Random-effect models were also utilised. MP knees were found to have a significant advantage over PS knees in terms of WOMAC score at the midterm follow up, and insignificant advantages over PS knees in terms of ROM and FJS at one and two years followup. Additionally, the PS knees demonstrated an in significantly higher Knee Society Score (KSS) at short and midterm follow up. In terms of ROM, KSS, OKS and FJS this meta-analysis suggests a non-significant advantages for the MP knee compared with the PS prothesis in the short term. The MP implant also

Conflict of Interest: The authors declare that they have no conflict of interest.

Funding: There is no funding source.

Ethical approval: This article does not contain any studies with human participants or animals performed by any of the authors. showed a significantly superior WOMAC score at short-term follow-up. An extended follow-up period is required to evaluate whether the MP knee is superior than the PS in the long-term.

Keywords: Medial pivot; posterior stabilised; total knee arthroplasty; meta-analysis.

Mr. Hany Elbardesy ${ }^{1}$, MSc, FRCS(Orth), FEBOT, SICOT Diploma

- Dr Hazem M Salamah ${ }^{2}$

- Dr. André McLeod ${ }^{1}$, Orthopaedic Senior House Officer

- Dr Pawan Kumar Thada ${ }^{3}$

- Dr Eman Reda Mohammed ${ }^{4}$

- Fatima Alzhra Hanifa ${ }^{5}$

- Mostafa Roshdy ${ }^{6}$

- Mr. Shane Guercin ${ }^{1}$, Consultant of Trauma and Orthopaedic Surgery

${ }^{1}$ Department of trauma and orthopaedic, Cork University Hospital, Wilton, Cork, Ireland.

${ }^{2}$ School of Medicine, Zagazig University, Zagazig, Egypt. ${ }^{3}$ University of Health Sciences, Pakistan.

${ }^{4}$ Kasr-Alainy Medical school, Cairo University, Egypt. ${ }^{5}$ Faculty of Medicine, University of Aleppo, Aleppo, Syria. ${ }^{6}$ Al-Azhar University Faculty of Medicine: Cairo, Egypt.

Correspondence : Mr. Hany Elbardesy, MSc, FRCS(Orth), FEBOT, SICOT Diploma, Orthopaedic Registrar, Department of trauma and orthopaedic, Cork University Hospital, Wilton, Cork, Ireland.. Phone : +353214922000 , Fax : +353214921383, ORCID ID : https://orcid.org/0000-00018600-6339.

Email : elbardecy@hotmail.com

- 2021, Acta Orthopædica Belgica. 


\section{INTRODUCTION}

Total knee arthroplasty (TKA) is commonly performed surgery for management of end stage knee osteoarthritis (OA) with a prosthesis survival rate of $92 \%$ at 16 years (1). Despite the high survival rates of the implants, only $82 \%$ to $89 \%$ of the patients are satisfied with the overall outcome (2). Researchers of the kinematics of the native knee have advocated for altering the joint congruity of TKA implants in order to replicate the medial pivoting of the native knee (3). The inability to mimic the normal physiological knee kinematics may play an important role in patient dissatisfaction postoperatively (4-5). Modern bicondylar TKAs are often designed using a dished bearing surface or a campost mechanism as these concepts help to recreate tibiofemoral rollback and stability in the sagittal plane (6-8). Posterior stabilized (PS) TKAs utilise the cam-post mechanism whereby the cam-post articulates with the tibial post improving femoral rollback and at the same time providing sagittal stability (8-12). Despite this it is associated with central tibial impingement and increased bleeding intra-operatively secondary to the larger osteotomy (13). Moreover, these alteration in the normal knee kinematic might contribute to the knee function and the survival of the prothesis. Additionally, the contradictory anterior femoral translation might negatively affect the extensor mechanism of the knee (14). The medial pivoting mechanism in native knees stems from external rotation of the femur in relation to the tibia during the knee movement from full extension to mid-flexion (15). MP TKAs are fixed bearing pivoting design prostheses. They designed with a congruent medial compartment to constrain the translation in the sagittal plane medially, and a non-congruent lateral compartment to allow femoral rollback on the lateral side of the knee (16). A systematic review performed by Young et al. (17) was unable to establish any clinical advantage of MP over non-MP TKAs due to the limited number of studies included. To our knowledge no published systematic reviews have comparatively evaluated MP and PS TKAs in the context of Patient Reporting Outcome Measures (PROMs) and post-operative ROM. This study aims to use meta-analysis and systematic review techniques to evaluate the ROM and PROM associated with MP and PS TKAs.

\section{MATERIALS AND METHODS}

We performed this study following both the Preferred Reporting Items for Systematic Reviews and Meta-analyses Statement (PRISMA) and the Cochrane Handbook for systematic reviews and meta-analysis (18). We conducted an initial search using MEDLINE-OVID, Web of Science, PubMed, EMBASE-OVID, Google Scholar and Cochrane Library. Grey and unpublished literature were also explored by searching: Grey Matters BIOSIS Previews, International Clinical Trial Registry, UK Clinical Trials Gateway, ClinicalTrials. gov, Networked Digital Library of Theses and Dissertations, UK Clinical Research Network Study Portfolio, Open Grey and Grey Literature Report. We used the following keywords and their combinations: medial pivot, posterior stabilised, and total knee arthroplasty. Articles published up to January 2021 were included in our literature search and were limited to studies in human subjects published in any language. Additionally, we crossreferenced the bibliographies of retrieved articles and review papers to ensure that we captured all relevant studies.

We included all comparative studies (retrospective/prospective cohorts, RCTs) involving patients undergoing unilateral or bilateral TKAs which were of MP or PS design, and where outcomes were compared between the two designs. We excluded cadaveric, in vitro, or single arm studies. Conference abstracts, letters to editor, reviews were also excluded.

Five authors independently screened all titles and abstracts identified by the initial search to assess their eligibility for inclusion. We then did a full screening of each manuscript and conducted a final assessment of the eligibility for all included studies. The same reviewers performed the data extraction. Any discrepancies found after data collection were resolved by discussion between all reviewers. The collected information included first author, year, country, study design, study length, number of participants, age, gender, and Body Mass Index (BMI). 


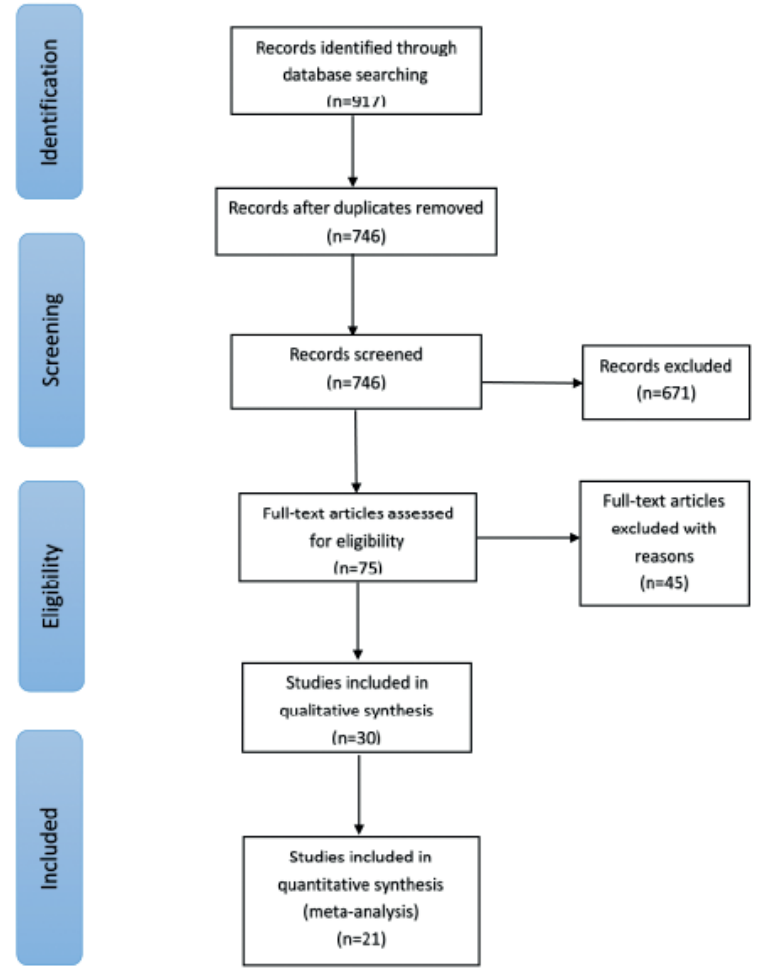

Figure 1. - Preferred reporting items for systematic reviews and meta-analyses (PRISMA) flow chart.

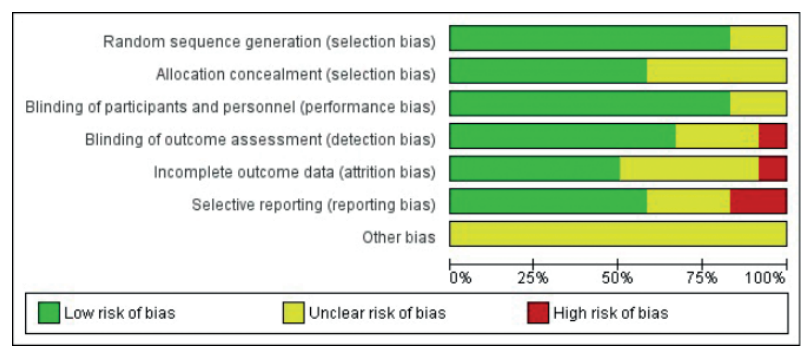

Figure 2. - Risk of bias graph: Review authors' judgements about each risk of bias item presented as percentages across all included studies.

We assessed the risk of bias for RCTs by using the Cochrane risk of bias criteria (Fig. 2 and 3) (18) and the non randomized cohort studies using NewcastleOttawa Scale (19). Five reviewers independently crossed-checked the quality of the included studies. Disagreements were resolved through discussion.

Twelve RCTs (14,20-30), were assessed for potential bias using Cochrane risk of bias tool. Randomization was performed in ten studies (20-25,2730 ), and use of randomization was unclear in two

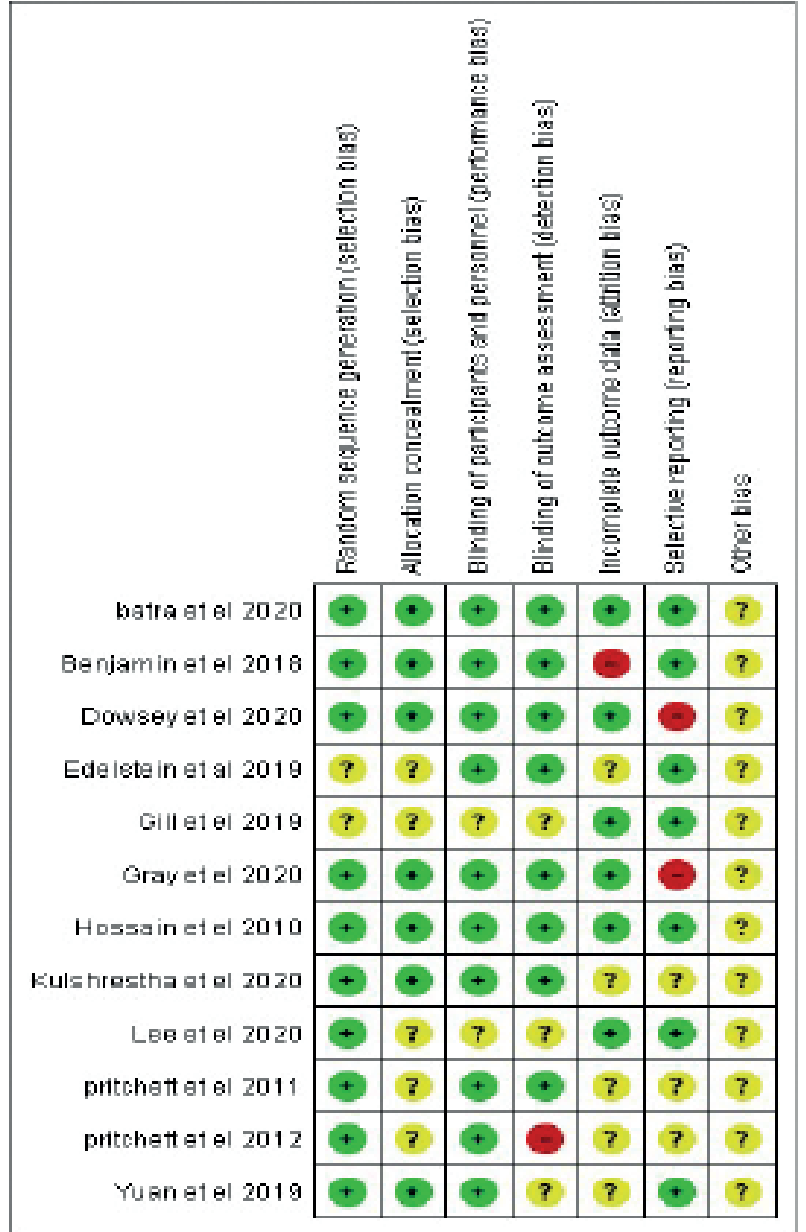

Figure 3. - Risk of bias summary: Review authors' judgements about each risk of bias item for each included study.

studies $(25,26)$. Use of patient blinding was unclear in two studies $(23,25)$. Quality assessment of 18 nonrandomized cohort studies using Newcastle-Ottawa Scale showed that 13 studies were of high quality while five studies (31-35) were of moderate quality (Table 3).

The primary outcome of interest was ROM. Secondary outcomes were Knee Society Score(KSS) (36), Forgotten Joint Score (FJS) (37), Western Ontario and McMaster Universities Osteoarthritis Index (WOMAC) (38), and Oxford Knee Score (OKS) (39).

We conducted a statistical analysis by using Review Manager(RevMan), version 5.3 (The Nordic Cochrane Centre, The Cochrane Collaboration, 2009, Copenhagen, Denmark) (40). Heterogeneity 
668 H. ElbARDESY, H.M. SALAMAH, A. MCLEOD, P.K. THADA, E.R. MOHAMMED, F.A. HANIFA, M. ROSHDY, S. GUERIN

Table 1. - Patient demographics by study

\begin{tabular}{|c|c|c|c|c|c|c|}
\hline \multirow[t]{2}{*}{ Study ID } & \multicolumn{2}{|c|}{ Age (year) } & \multicolumn{2}{|c|}{ BMI (kg/m2) } & \multicolumn{2}{|c|}{ Gender (female percentage) } \\
\hline & MP & PS & MP & PS & MP & PS \\
\hline Samy et al. 2017 & $64.4 \pm 10.5$ & $66.7 \pm 8.61$ & $29.7 \pm 5.24$ & $31.3 \pm 8.2$ & $47(61.8 \%)$ & $54(61.4 \%)$ \\
\hline Papagiannis et al. 2016 & $70.25 \pm 1.96$ & $72.92 \pm 1.46$ & NA & & NA & \\
\hline Lin et al. 2020 & $70.38 \pm 6.37$ & $70.98 \pm 7.29$ & NA & & $33(32 \%)$ & $222(82 \%)$ \\
\hline Kulshrestha et al. 2020 & $63.8 \pm 6.8$ & $65.97 \pm 6.7$ & $27.34 \pm 5.1$ & $26.64 \pm 4.3$ & $29(72.5 \%)$ & $23(57.5 \%)$ \\
\hline Lee et al. 2020 & $70 \pm 7$ & $70 \pm 7$ & $27.4 \pm 4$ & $27.4 \pm 4$ & $32(69.6 \%)$ & $32(69.6 \%)$ \\
\hline Hossain et al. 2011 & $72.5 \pm 9.7$ & $68.9 \pm 12.1$ & $28.9 \pm 6.2$ & $29.5 \pm 8.1$ & $31(77.5 \%)$ & $22(55 \%)$ \\
\hline Gray et al. 2020 & $67.3 \pm 6.44$ & $66.8 \pm 7.34$ & $33.8 \pm 4.6$ & $30.9 \pm 4.5$ & $14(53.85 \%)$ & $9(39.13 \%)$ \\
\hline Gill et al. 2019 & $68.86 \pm 2.67$ & $68.6 \pm 2.09$ & NA & & $22(62.9 \%)$ & $24(68.6 \%)$ \\
\hline Esposito et al. 2020 & $73.3 \pm 3.5$ & $70.5 \pm 4.7$ & $31 \pm 3$ & $30.9 \pm 3.6$ & $11(55 \%)$ & $8(40 \%)$ \\
\hline Edelstein et al 2019 & $67 \pm 8$ & $64 \pm 7$ & $32.8 \pm 5.82$ & $34.2 \pm 5.81$ & $18(72 \%)$ & $15(60 \%)$ \\
\hline Dowsey et al. 2020 & $66 \pm 6.8$ & $65.7 \pm 7.7$ & $32.5 \pm 3.6$ & $30.7 \pm 3.8$ & $15(51.7 \%)$ & $11(42.3 \%)$ \\
\hline Benjamin et al. 2018 & $62.4(54-71)$ & $64.8(58-73)$ & NA & & $4(40 \%)$ & $3(30 \%)$ \\
\hline Bae et al. 2016 & $66.7 \pm 7.1$ & $66.7 \pm 6.5$ & $26.4 \pm 3.2$ & $25.9 \pm 4.4$ & $120(96.8 \%)$ & $136(98.6 \%)$ \\
\hline Anderson et al. 2002 & $70(47-84)$ & $69(38-89)$ & NA & & $11(55 \%)$ & $14(70 \%)$ \\
\hline Minoda et al. 2003 & $67 \pm 2.3$ & $63.8 \pm 2.1$ & NA & & NA & \\
\hline Indelli et al. 2018 & $67.3(53-77)$ & $67.6(46-86)$ & $34.6(23.7-42.4)$ & $34.4(25.7-53.8)$ & $3(7 \%)$ & $4(8 \%)$ \\
\hline Wautier et al. 2016 & $72 \pm 11$ & $69.5 \pm 9.5$ & $33 \pm 5$ & $28 \pm 4.8$ & $6(60 \%)$ & $13(65 \%)$ \\
\hline Beach et al. 2019 & $66.7 \pm 5.6$ & $69.1 \pm 6.4$ & $32.3 \pm 3.9$ & $30.1 \pm 5.4$ & NR & \\
\hline Vanduren et al. 2007 & $75.2(68-78)$ & $72.4(64-78)$ & NA & & NR & \\
\hline Zhang et al. 2020 & $67.5 \pm 6.5$ & $65.4 \pm 6.2$ & $27.3 \pm 3$ & $27.6 \pm 3$ & $74(76 \%)$ & $80(73.4 \%)$ \\
\hline Yuan et al. 2019 & $69.43 \pm 5.97$ & $69.63 \pm 5.72$ & $27.81 \pm 5.17$ & $27.59 \pm 4.86$ & $26(54 \%)$ & $28(55 \%)$ \\
\hline Shi et al. 2020 & $74.5 \pm 6.97$ & $75.4 \pm 5.7$ & $27.89 \pm 3.65$ & $27.43 \pm 3.51$ & $228(78.6 \%)$ & $169(71.3 \%)$ \\
\hline Shakespeare et al. 2006 & 76 & 78 & NA & & $49 \%$ & $52 \%$ \\
\hline Schütz et al. 2019 & $68.8 \pm 9.9$ & $69 \pm 6.5$ & $25.4 \pm 3.7$ & $27.6 \pm 3.5$ & $8(80 \%)$ & $5(50 \%)$ \\
\hline Pritchett et al. 2012 & 66.4 & 69.6 & NA & & NA & \\
\hline Pritchett et al. 2011 & NA & & NA & & NA & \\
\hline Hosseininasab et al. 2019 & $68.8 \pm 9.9$ & $69 \pm 6.5$ & $25.4 \pm 3.7$ & $27.6 \pm 3.5$ & $8(80 \%)$ & $5(50 \%)$ \\
\hline Batra et al. 2020 & $61.7 \pm 6.88$ & $61.7 \pm 6.88$ & $28.3 \pm 3.4$ & $28.3 \pm 3.4$ & $42(79.2 \%)$ & $42(79.2 \%)$ \\
\hline Wang et al. 2021 & $66.92 \pm 5.60$ & $67.15 \pm 6.01$ & $27.74 \pm 4.63$ & $27.90 \pm 4.39$ & $102(81 \%)$ & $104(82.5 \%)$ \\
\hline Bianchi et al. 2021 & $\begin{array}{c}72 \text { (CI95\%: } \\
68-76)\end{array}$ & $\begin{array}{c}71 \text { (CI95\%: } \\
69-74)\end{array}$ & NA & & $8(50 \%)$ & $7(43.75 \%)$ \\
\hline
\end{tabular}

SD: standard deviation, NA: Not applicable, BMI: Body Mass Index.

between studies was assessed by the $\mathrm{I}^{2}$ statistic and a $\mathrm{c}^{2}$ of $<0.05$ was used to define the significance of the heterogeneity among the included studies. Ranges of $0-24 \%, 25-74 \%$ and $75-100 \%$ were used to define minor, moderate and major heterogeneity respectively (18). Mean differences and standard deviations (SDs) were used for continuous variables. We used the Mantel-Haenszel random-effects model in our meta-analysis. We illustrated the results using forest plots, which used a 95\% confidence interval (CI) for each study and a cumulative weighted Mean Differences (MD) for all the included studies (18).

\section{RESULTS}

Our literature search returned 746 articles after removal of duplicates. Title and abstract screening revealed 75 articles that were eligible for full text screening. Forty-five articles were subsequently excluded for not meeting selection criteria leaving 30 articles that were included for qualitative review. Twenty-one of these articles were included in the meta-analysis. A flow chart demonstrating the study selection process is provided (Fig 1). Twelve studies were randomized clinical trials (RCTs), five were prospective cohort studies and thirteen 


\begin{tabular}{|c|c|c|c|c|c|c|c|c|}
\hline 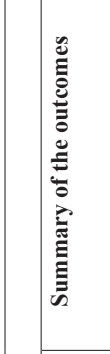 & 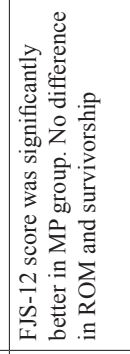 & 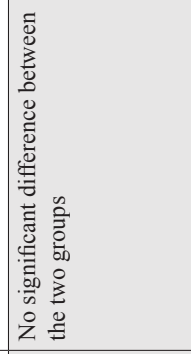 & 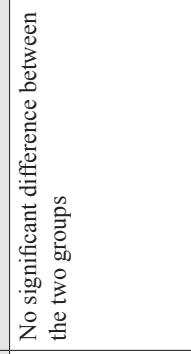 & 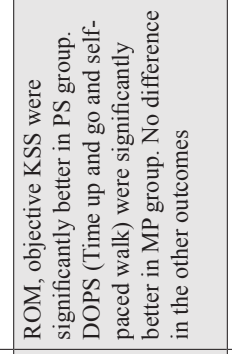 & 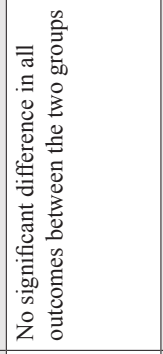 & 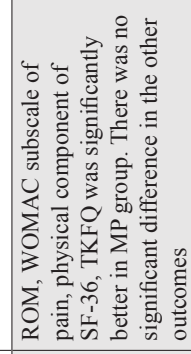 & 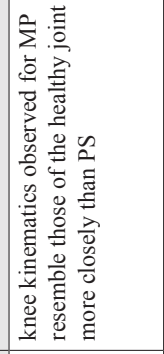 & 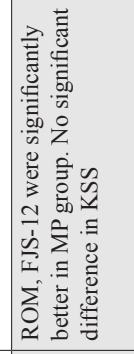 \\
\hline 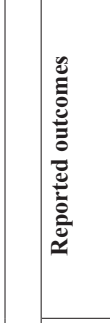 & 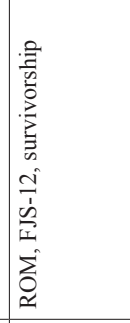 & 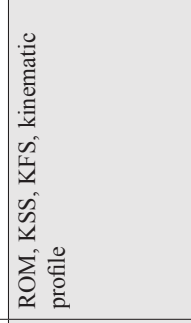 & 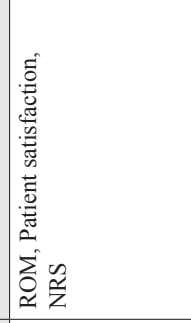 & 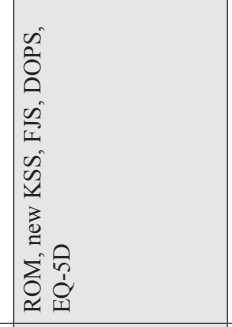 & 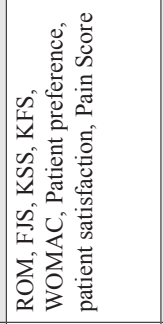 & 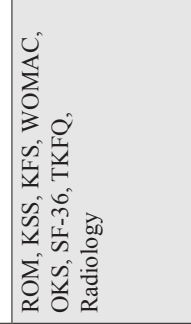 & 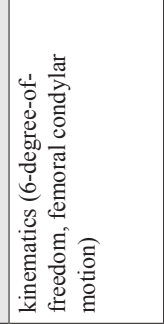 & 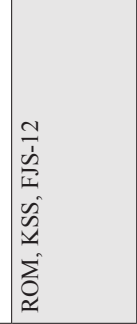 \\
\hline 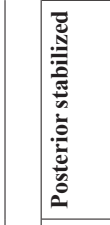 & 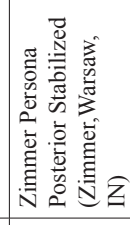 & 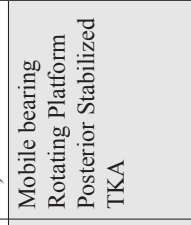 & 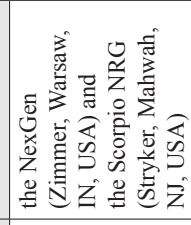 & 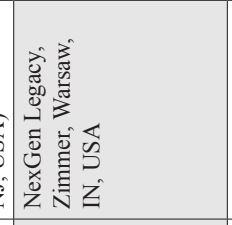 & 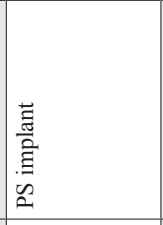 & 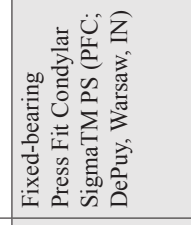 & 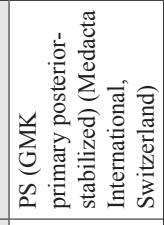 & 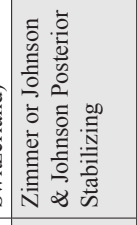 \\
\hline 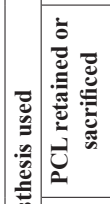 & $\tilde{z}$ & $\tilde{z}$ & $\tilde{z}$ & 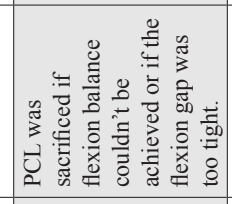 & $\tilde{z}$ & 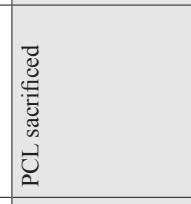 & 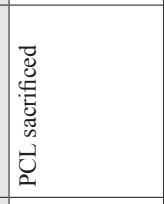 & 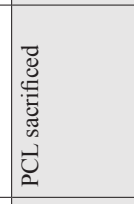 \\
\hline 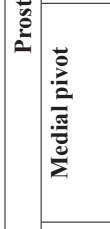 & 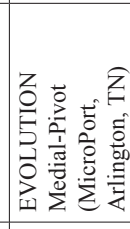 & 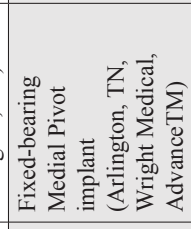 & 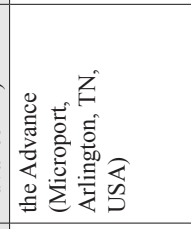 & 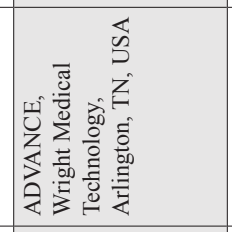 & 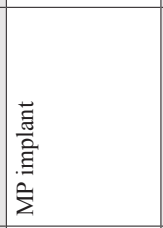 & 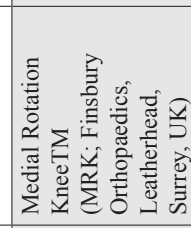 & 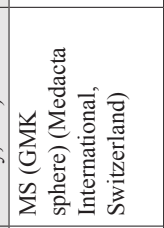 & 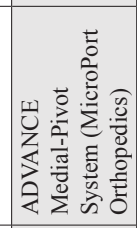 \\
\hline 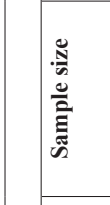 & 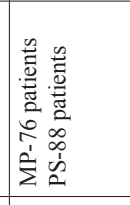 & 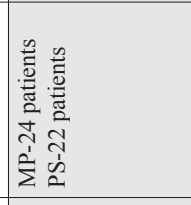 & 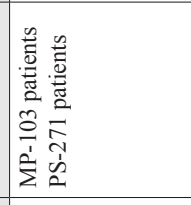 & 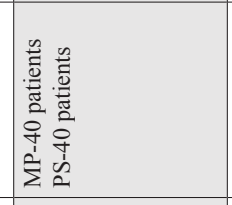 & 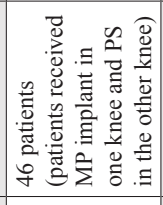 & 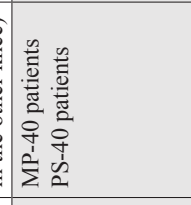 & 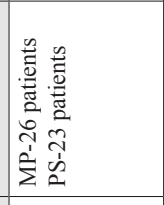 & 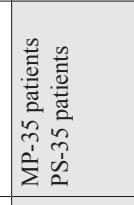 \\
\hline 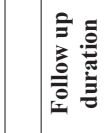 & 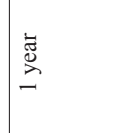 & 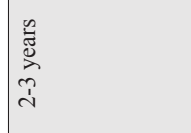 & 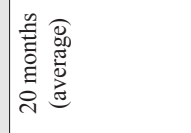 & 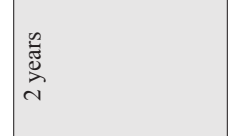 & 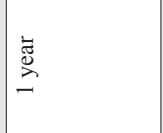 & 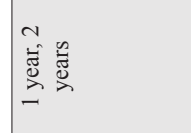 & 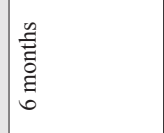 & 岂 \\
\hline 咅 & 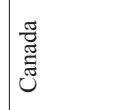 & 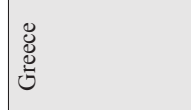 & 爵 & 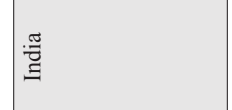 & 営 & כ & 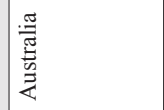 & 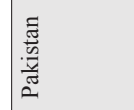 \\
\hline 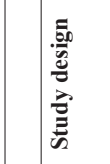 & 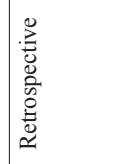 & 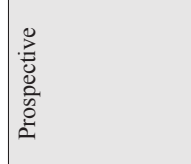 & 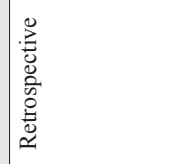 & $\underset{\approx}{\tilde{q}}$ & : & $\underset{\approx}{E}$ & 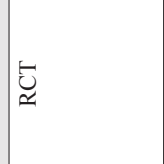 & $\underset{\approx}{\tilde{\approx}}$ \\
\hline 焉 & 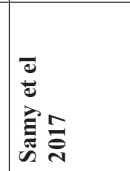 & 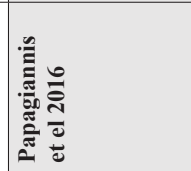 & 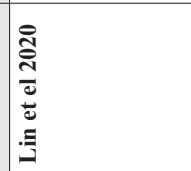 & 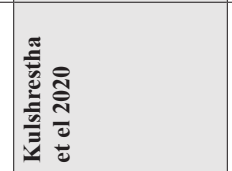 & 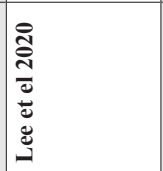 & 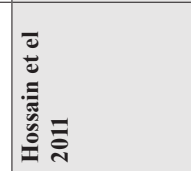 & 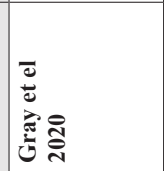 & 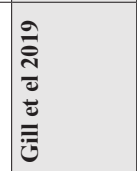 \\
\hline
\end{tabular}


670 H. ELBARDESY, H.M. SALAMAH, A. MCLEOD, P.K. THADA, E.R. MOHAMMED, F.A. HANIFA, M. ROSHDY, S. GUERIN

\begin{tabular}{|c|c|c|c|c|c|c|c|c|}
\hline 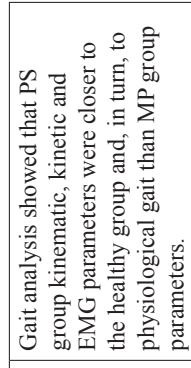 & 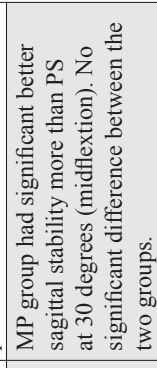 & 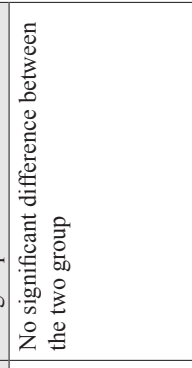 & 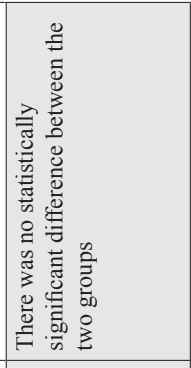 & 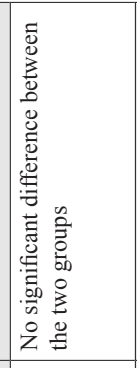 & 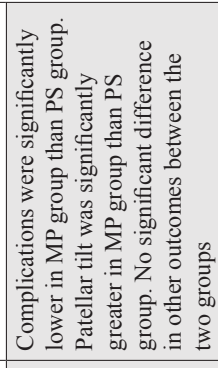 & 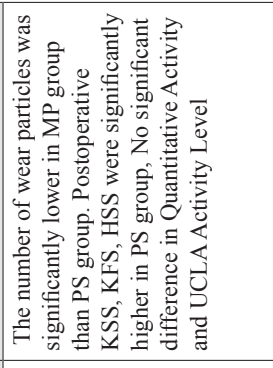 & 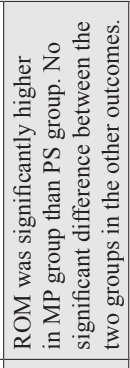 & 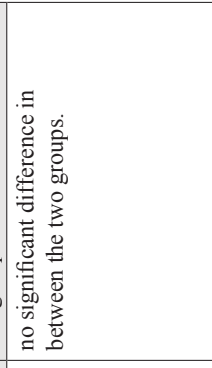 \\
\hline 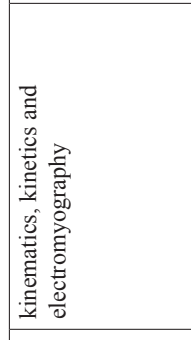 & 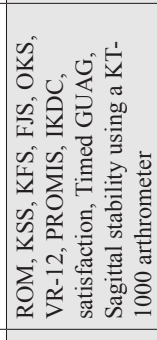 & 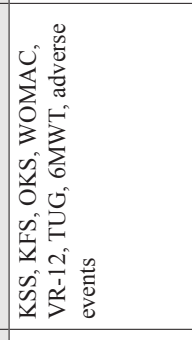 & 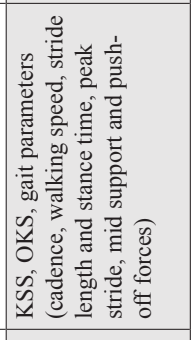 & 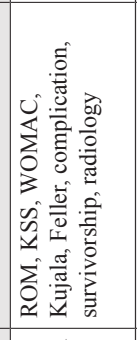 & 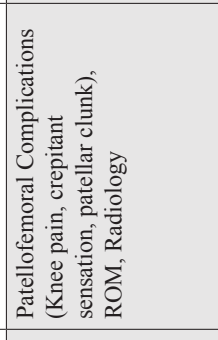 & 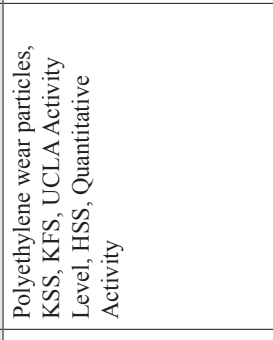 & 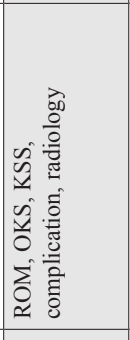 & 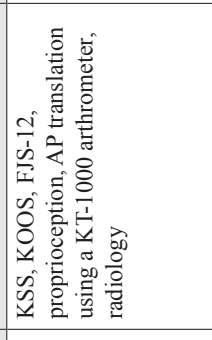 \\
\hline 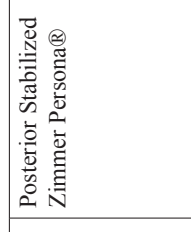 & 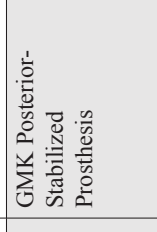 & 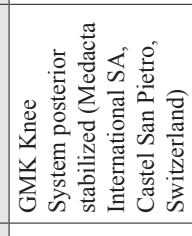 & 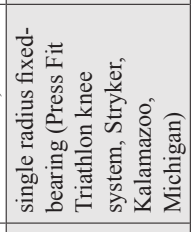 & 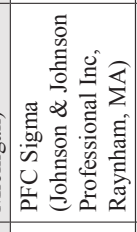 & 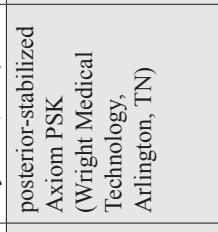 & 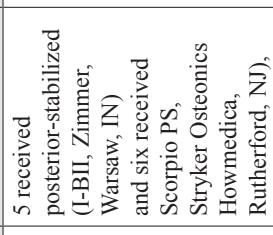 & 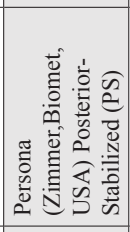 & 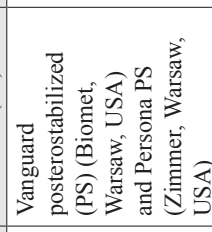 \\
\hline 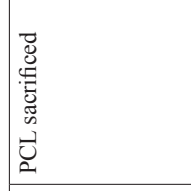 & & $\tilde{z}$ & 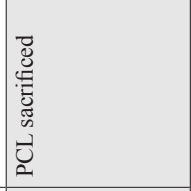 & 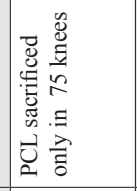 & 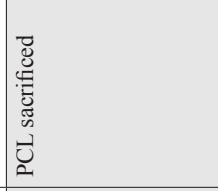 & 䒘 & 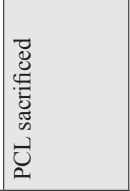 & $\tilde{z}$ \\
\hline 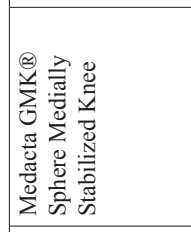 & 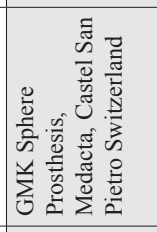 & 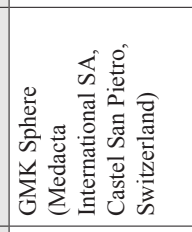 & 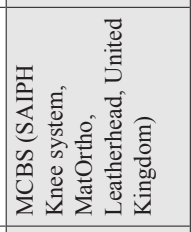 & 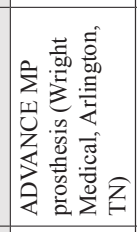 & 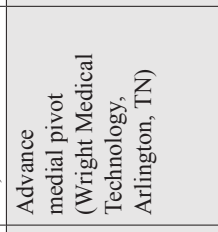 & 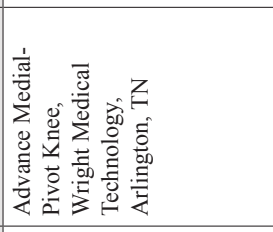 & 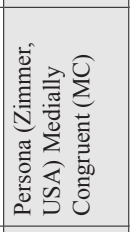 & 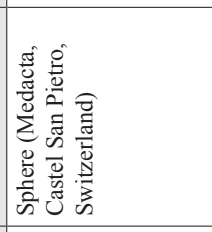 \\
\hline 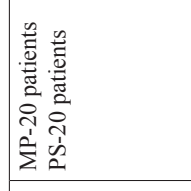 & 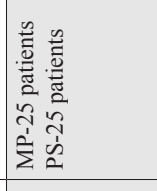 & 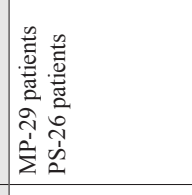 & 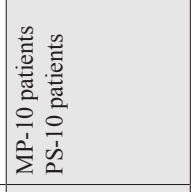 & 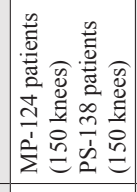 & 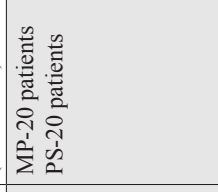 & 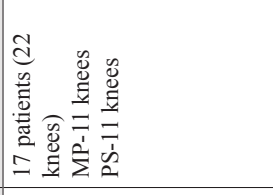 & 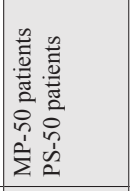 & 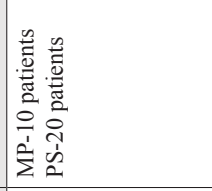 \\
\hline 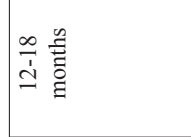 & 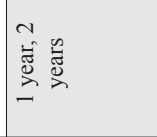 & 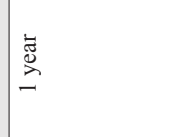 & 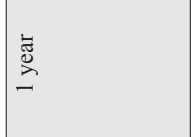 & 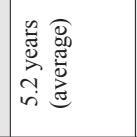 & 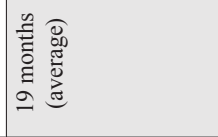 & 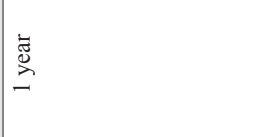 & 崀 & 氦 \\
\hline 忞 & 厽 & 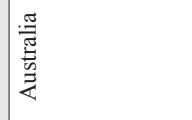 & $\breve{s}$ & 莺 & 芯 & 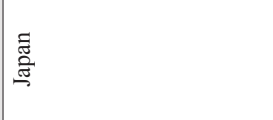 & 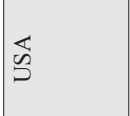 & 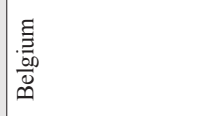 \\
\hline 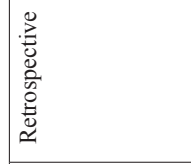 & $\underset{\simeq}{t}$ & $\begin{array}{l}\vec{U} \\
\dddot{\simeq}\end{array}$ & $\underset{\simeq}{\underline{y}}$ & 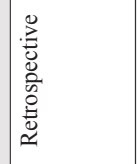 & 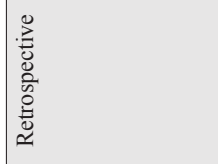 & 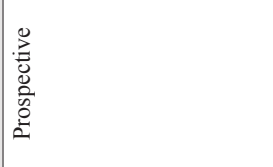 & 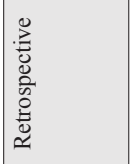 & 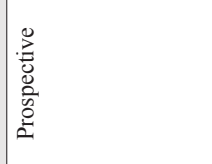 \\
\hline 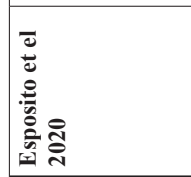 & 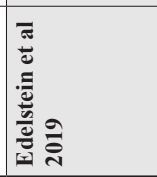 & 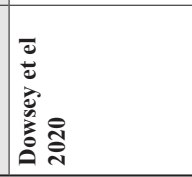 & 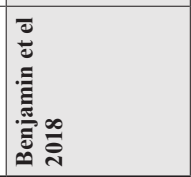 & 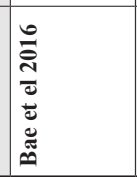 & 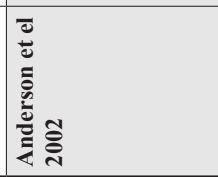 & 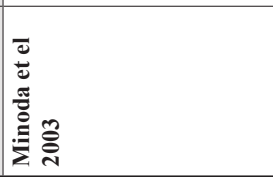 & 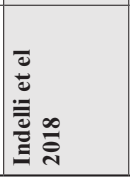 & 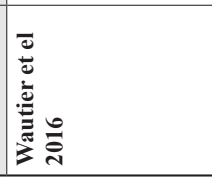 \\
\hline
\end{tabular}




\begin{tabular}{|c|c|c|c|c|c|c|c|c|c|}
\hline 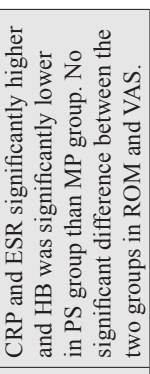 & 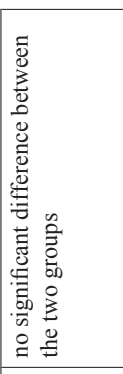 & 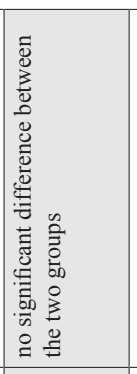 & 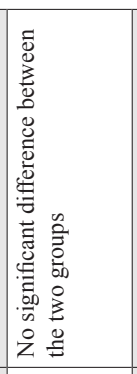 & 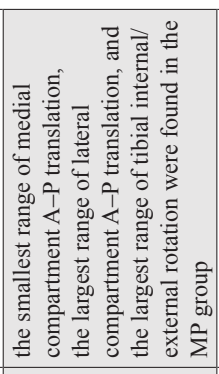 & 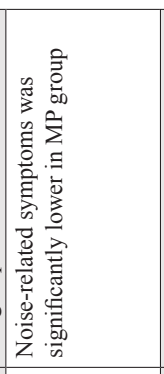 & 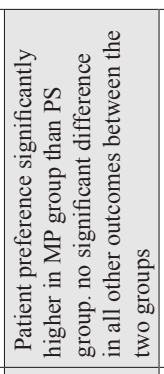 & 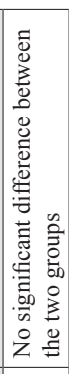 & 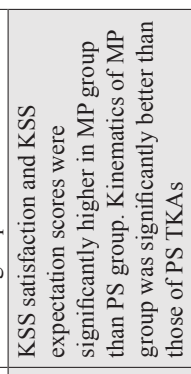 & 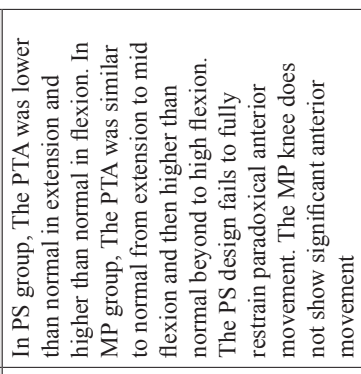 \\
\hline 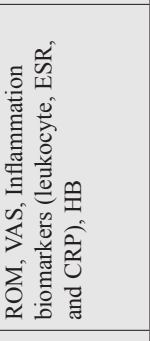 & 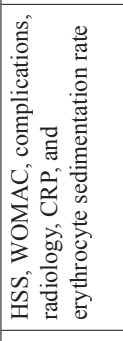 & 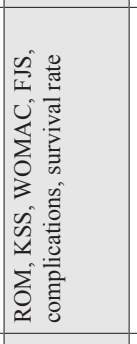 & $\begin{array}{l}\sum_{0} \\
\approx\end{array}$ & 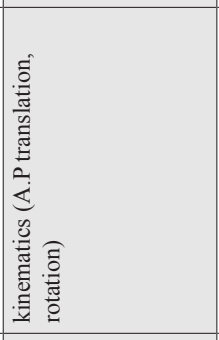 & 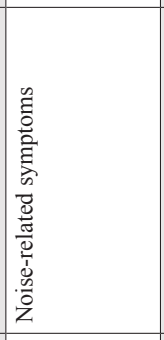 & 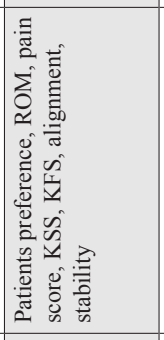 & 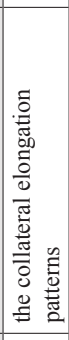 & 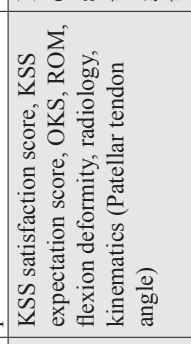 & 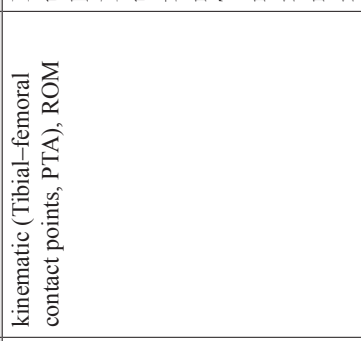 \\
\hline 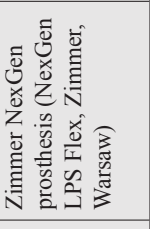 & 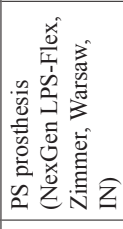 & 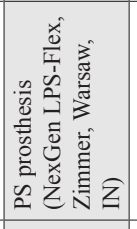 & 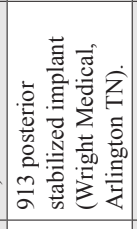 & 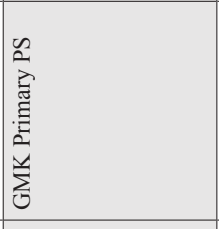 & 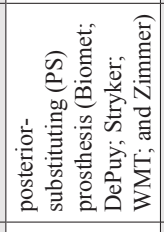 & 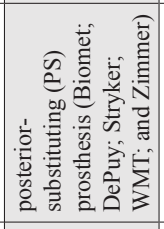 & 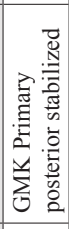 & 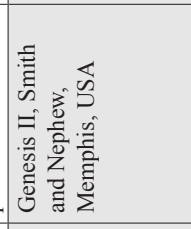 & $\begin{array}{l}n \\
0 \\
0 \\
0 \\
0 \\
0 \\
0\end{array}$ \\
\hline$\tilde{z}$ & & $\tilde{z}$ & $\tilde{z}$ & $\tilde{z}$ & 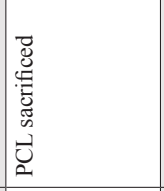 & 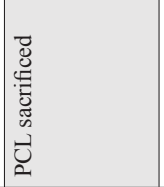 & $\frac{\tilde{z}}{z}$ & 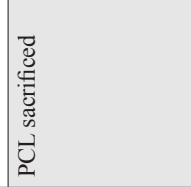 & z̃ \\
\hline 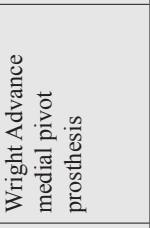 & 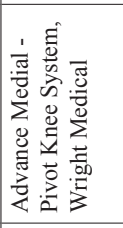 & 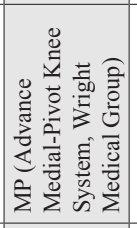 & 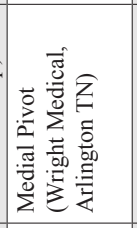 & 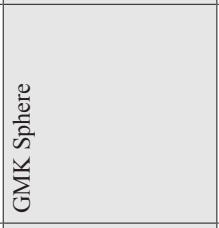 & 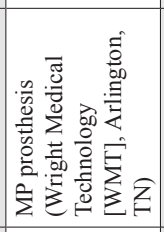 & 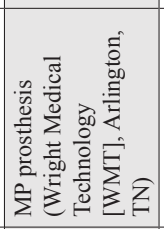 & 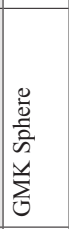 & 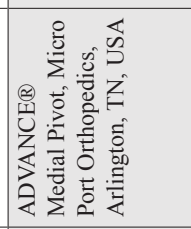 & 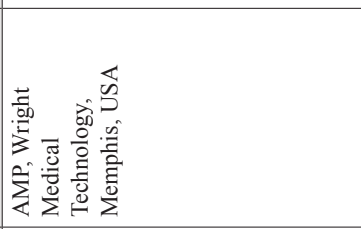 \\
\hline 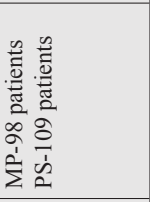 & 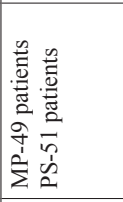 & 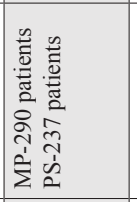 & 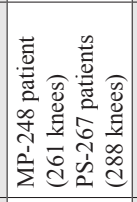 & 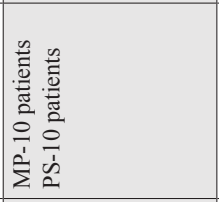 & 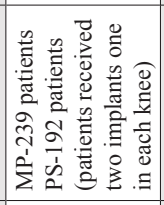 & 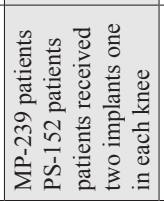 & 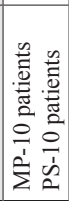 & 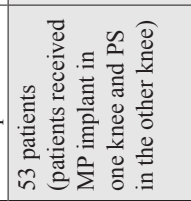 & 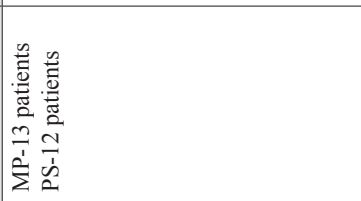 \\
\hline 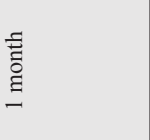 & 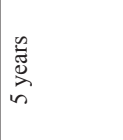 & $\begin{array}{l}0 \\
\frac{n}{\tilde{E}} \\
\widehat{2} \\
6\end{array}$ & 总 & 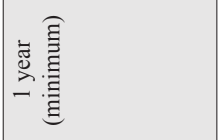 & 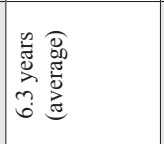 & 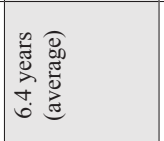 & 总 & 产 & 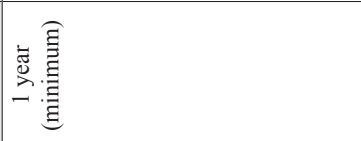 \\
\hline : & 葋 & 氶 & $\breve{J}$ & 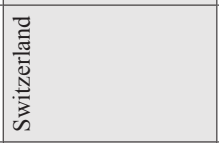 & 崩 & 占 & 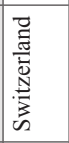 & 舀 & $\breve{s}$ \\
\hline 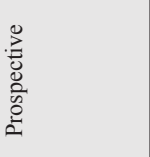 & $\underset{\approx}{5}$ & 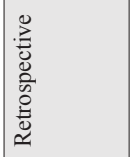 & 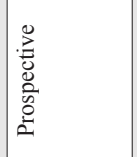 & 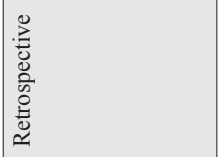 & $\underset{\approx}{E}$ & $\approx$ & 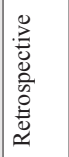 & $\underline{\approx}$ & 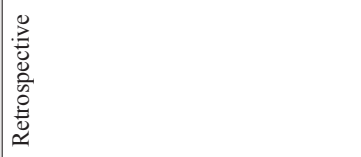 \\
\hline 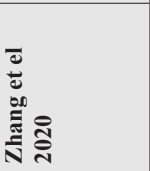 & 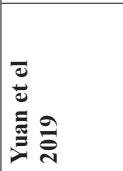 & 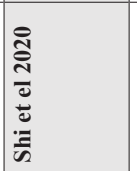 & 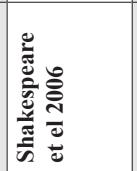 & 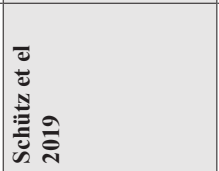 & 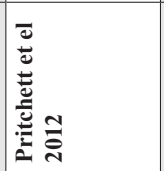 & 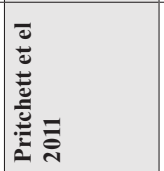 & 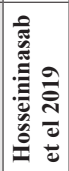 & 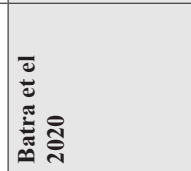 & 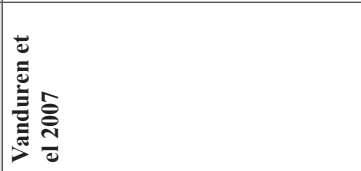 \\
\hline
\end{tabular}




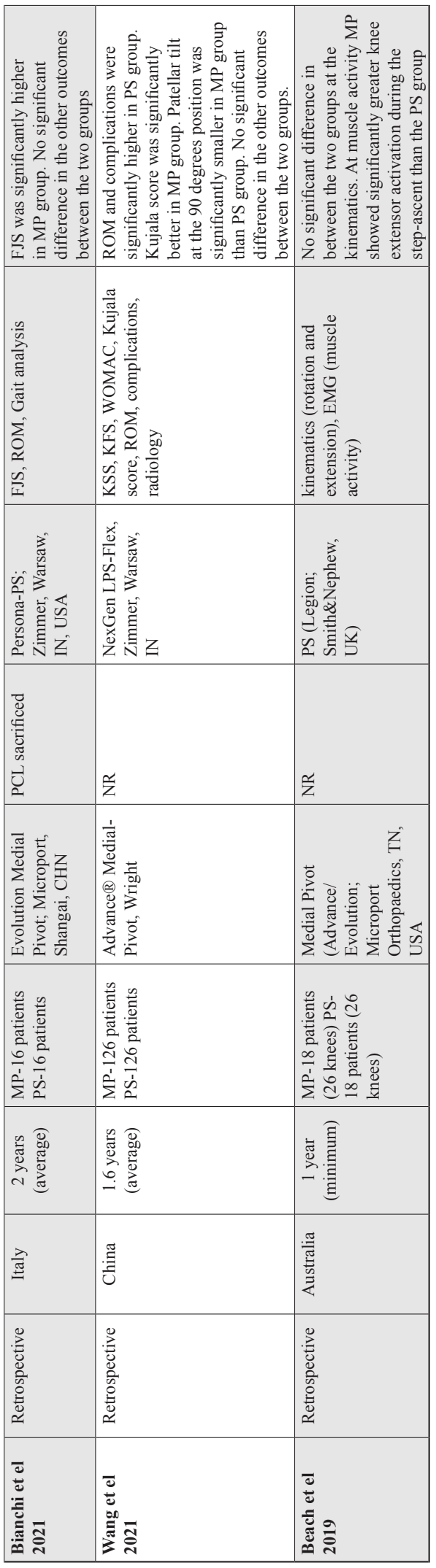

were retrospective cohort studies. A summary of the characteristics of included studies is provided (Table 2).

Our review included 3837 knees (1855 in MP group, 1982 in PS group). The subjects in the MP group had an average age of 68.7 years $( \pm 3.7$ years $)$, of which 884 out of 1855 patients $(61.54 \%)$ were female, with an average body mass index of 29.68 $\mathrm{kg} / \mathrm{m}^{2}( \pm 2.8)$. The PS cohort had a similar patient distribution with an average age of 68.5 years (range: ( \pm 3.3 years), of which 1025/1982 (58.88\%) were female, with an average body mass index of $29.24 \mathrm{~kg} / \mathrm{m} 2( \pm 6.58)$. A summary of the patient demographics of included studies is provided (Table 1).

Our meta-analysis comparatively assessed the ROM, KSS (total and functional), and WOMAC scores at one year, two years and midterm (5-8 years) follow up. We also assessed FJS and OKS at one and two years follow-up (41).

Overall, six studies assessing 1142 knees reported on postoperative ROM after one year. 546 of these were of MP design while 596 were of PS design. The MP group reported an insignificantly higher ROM. The heterogeneity analysis demonstrated major statistical evidence for variation within the study $\left(\mathrm{I}^{2}\right.$ $=86 \%)$. The cumulative MD was significant at 3.43 (95\% CI, -0.53-7.39; $\mathrm{P}<0.09$ ) (Fig 4).

Nine studies including 1067 knees reported on postoperative ROM after two years. In this group there were $450 \mathrm{MP}$ and 617 PS TKAs. Once again, the MP cohort reported insignificantly superior ROM compared to the PS group. The heterogeneity analysis demonstrated major statistical evidence for variation within the study $\left(\mathrm{I}^{2}=92 \%\right)$. The cumulative MD was significant at 1.92 (95\% CI, -1.18-5.03; P < 0.22) (Fig 4).

Three studies reported on ROM at midterm follow-up with a total of 933 knees assessed. Out of these, 493 were MP knees and 440 were PS knees. Heterogeneity analysis demonstrated moderate evidence for variation within the study $\left(\mathrm{I}^{2}\right.$ $=66 \%$ ). Data analysed by the random-effects model suggested no significant difference for the midterm ROM between the two cohorts. The cumulative MD was significant at 0.90 (95\% CI, 3.37-5.17; P $<0.96$ ) (Fig 4). 
Table 3. - Newcastle-Ottawa Scale (NOS) for assessing the quality of Cohort studies

\begin{tabular}{|c|c|c|c|c|c|c|c|c|c|}
\hline \multirow[t]{2}{*}{ Study } & \multicolumn{4}{|c|}{ Selection } & \multirow{2}{*}{$\begin{array}{c}\text { Comparability } \\
\text { Comparability of } \\
\text { Cohorts on the Basis } \\
\text { of the Design or } \\
\text { Analysis }\end{array}$} & \multicolumn{3}{|c|}{ Exposure } & \multirow{2}{*}{$\begin{array}{c}\text { Total } \\
\text { Total } \\
\text { number } \\
\text { of stars }\end{array}$} \\
\hline & $\begin{array}{l}\text { Representati- } \\
\text { veness of the } \\
\text { Exposed } \\
\text { Cohort }\end{array}$ & $\begin{array}{l}\text { Selection } \\
\text { of the Non- } \\
\text { Exposed } \\
\text { Cohort }\end{array}$ & $\begin{array}{l}\text { Ascertain- } \\
\text { ment of } \\
\text { Exposure }\end{array}$ & $\begin{array}{c}\text { Demonstration } \\
\text { That Outcome of } \\
\text { Interest Was Not } \\
\text { Present at Start of } \\
\text { Study }\end{array}$ & & $\begin{array}{l}\text { Assessment } \\
\text { of Outcome }\end{array}$ & $\begin{array}{l}\text { Was Follow-Up } \\
\text { Long Enough } \\
\text { for Outcomes to } \\
\text { Occur }\end{array}$ & $\begin{array}{l}\text { Adequacy } \\
\text { of Follow } \\
\text { Up of } \\
\text { Cohorts }\end{array}$ & \\
\hline Samy et al. 2017 & * & * & $*$ & $*$ & $*$ & $*$ & $*$ & 0 & 7 \\
\hline Lin et al. 2020 & 0 & $*$ & $*$ & $*$ & $*$ & $*$ & $*$ & 0 & 6 \\
\hline Bae et al. 2016 & * & $*$ & $*$ & * & $* *$ & * & $*$ & $*$ & 9 \\
\hline Anderson et al. 2002 & $*$ & $*$ & $*$ & $*$ & $*$ & $*$ & $*$ & $*$ & 8 \\
\hline Indelli et al. 2018 & 0 & $*$ & $*$ & $*$ & $* *$ & $*$ & $*$ & $*$ & 8 \\
\hline Beach et al. 2019 & $*$ & $*$ & $*$ & $*$ & $* *$ & $*$ & $*$ & $*$ & 9 \\
\hline Shi et al. 2020 & * & $*$ & $*$ & $*$ & $* *$ & $*$ & $*$ & 0 & 8 \\
\hline Schütz et al. 2019 & * & $*$ & $*$ & * & $* *$ & $*$ & $*$ & $*$ & 9 \\
\hline $\begin{array}{l}\text { Hosseininasab et al. } \\
2019\end{array}$ & $*$ & $*$ & $*$ & $*$ & $* *$ & $*$ & * & 0 & 8 \\
\hline Vanduren et al. 2007 & $*$ & $*$ & $*$ & $*$ & $* *$ & $*$ & $*$ & 0 & 8 \\
\hline Papagiannis et al. 2016 & * & * & $*$ & * & $*$ & $*$ & $*$ & 0 & 7 \\
\hline Esposito et al. 2020 & $*$ & $*$ & $*$ & $*$ & $* *$ & $*$ & $*$ & $*$ & 9 \\
\hline Minoda et al. 2003 & $*$ & $*$ & $*$ & $*$ & $* *$ & $*$ & $*$ & $*$ & 9 \\
\hline Zhang et al. 2020 & $*$ & $*$ & $*$ & $*$ & $*$ & $*$ & 0 & $*$ & 7 \\
\hline Shakespeare et al. 2006 & $*$ & $*$ & $*$ & $*$ & $*$ & $*$ & $*$ & 0 & 7 \\
\hline Wautier et al. 2016 & $*$ & $*$ & $*$ & $*$ & $* *$ & $*$ & $*$ & $*$ & 9 \\
\hline Wang et al. 2021 & $*$ & $*$ & $*$ & $*$ & $* *$ & $*$ & $*$ & * & 9 \\
\hline Bianchi et al. 2021 & $*$ & $*$ & $*$ & $*$ & $* *$ & $*$ & $*$ & $*$ & 9 \\
\hline
\end{tabular}

Seven studies reported on KSS at 1 year followup encompassing 349 knees. 178 reported on PS protheses and 171 on MP prostheses. PS knees were found to have an insignificantly higher KSS at one year compared with MP knees. The heterogeneity analysis demonstrated moderate statistical evidence for variation within the study $\left(\mathrm{I}^{2}=65 \%\right)$. The cumulative MD was significant at -0.43 (95\% CI, 4.05- 3.19: $\mathrm{P}<0.82$ ) (Fig. 5).

Five studies including a total of 521 knees reported on postoperative KSS after two years. Out of that number, 261 were MP TKAs and 260 were PS TKAs. The PS group reported a superior KSS to the MP group. The heterogeneity analysis demonstrated high statistical evidence for variation within the study $\left(\mathrm{I}^{2}=91 \%\right)$. The cumulative MD was found to be significant at $-1.74(95 \% \mathrm{CI}, 1.45$ 4.94; $\mathrm{P}<0.29$ ) (Fig. 5).

Two studies reported on KSS at midterm followup with a total of 827 knees. This included $387 \mathrm{MP}$ prostheses and 440 PS prostheses. Heterogeneity analysis demonstrated high statistical evidence for variation within the study $\left(\mathrm{I}^{2}=95 \%\right)$. Data pooled by random-effects model suggested a significantly higher KSS for the PS knee. The cumulative MD was significant at $-0.90(95 \% \mathrm{CI}, 2.73-4.52$; $\mathrm{P}<$ 0.001) (Fig 5).

Five studies including 349 knees (148 PS; 151 MP) reported on KSS functional score after one year. PS knees demonstrated an insignificantly higher KSS functional score at one year follow-up. The heterogeneity analysis demonstrated moderate statistical evidence for variation within the study $\left(\mathrm{I}^{2}\right.$ $=58 \%)$. The cumulative MD was insignificant at 1.78 (95\% CI, 4.04-7.60: $\mathrm{P}=0.55$ ) (Fig. 6).

Four studies encompassing 451 knees (225 PS; $226 \mathrm{MP})$ reported on KSS functional score after two years. They reported a marginally higher nonsignificant functional KSS with the MP cohort versus the PS cohort. Heterogeneity analysis demonstrated low statistical evidence for variation within the study $\left(\mathrm{I}^{2}=42 \%\right)$. The cumulative MD was insignificant at $0.42(95 \% \mathrm{CI},-1.90-2.75$; P $=0.72$ ) (Fig 6).

Two studies reported on functional KSS at midterm follow-up encompassing a total of 827 
674 H. ElBARDESY, H.M. SALAMAH, A. MCLEOD, P.K. THADA, E.R. MOHAMMED, F.A. HANIFA, M. ROSHDY, S. GUERIN

\begin{tabular}{|c|c|c|c|c|c|c|c|c|c|c|c|c|}
\hline \multirow[b]{2}{*}{ Shudy er S.baroep } & \multicolumn{3}{|c|}{ MP } & \multicolumn{3}{|c|}{ Ps } & \multirow[b]{2}{*}{ Welatif } & \multirow{3}{*}{ 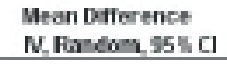 } & \multirow{2}{*}{\multicolumn{4}{|c|}{$\begin{array}{l}\text { Mean Daflerence } \\
\text { N. Fandom, } 95 \times \mathrm{Cl}\end{array}$}} \\
\hline & Mean & SD & Total & Mean & 50 & Total & & & & & & \\
\hline \multicolumn{12}{|l|}{ L1.1 from the years } & \\
\hline Echastein ef a 2018 & 1112 & 10.4 & 25 & 114.7 & 10.7 & 26 & $4.3 \%$ & $-3.60+930,230$ & & & & \\
\hline Hosasin atel 2010 & 1155 & 11.6 & 40 & 98.2 & 142 & 40 & 4.48 & $17.30[11.62,22.96$ & & & & \\
\hline Leget et 2020 & 100 & 12 & 46 & 110 & 14 & 46 & $4.7 \%$ & $-200+730,3.33$ & & & & \\
\hline $8 \mathrm{sm}$ ef el $201 \mathrm{~T}$ & 121.7 & 10.51 & 76 & 115.94 & 19.27 & 88 & $0.1 \%$ & $5.76[1.09,10.43]$ & & & & \\
\hline Shabespecre ot al 2006 & 111 & 14.62 & 261 & 109 & $14 B 2$ & 288 & 6.64 & $20010.45,4.49$ & & & & \\
\hline $\begin{array}{l}\text { Zhang of el } 2020 \\
\text { Subitutal } 1906 \mathrm{CD})\end{array}$ & 112 & 7.4 & $\begin{array}{r}98 \\
546\end{array}$ & 110.1 & 5.8 & $\begin{array}{l}108 \\
596\end{array}$ & $\begin{array}{l}7.0 \% \\
32.1 \%\end{array}$ & $\begin{array}{r}1.90 \text { p.07, } 3.73 \\
3.4310 .53,7.390\end{array}$ & & & & \\
\hline \multicolumn{13}{|c|}{ 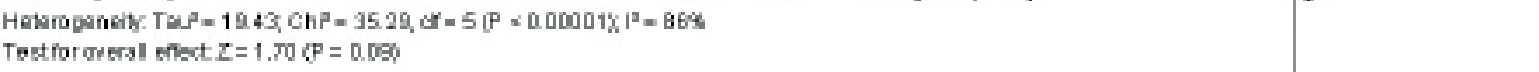 } \\
\hline \multicolumn{13}{|l|}{ 1.2. 100042 years } \\
\hline Anders on etel 2002 & 110 & 11.89 & 20 & 118 & 11.89 & 20 & $3.0 \%$ & $-3.00+10.37,4.37$ & & & & \\
\hline Bianchi of al 2021 & 118 & B. 4 & 16 & 112 & 938 & 16 & $4.1 \%$ & B. $00+0.17,12.17$ & & & & \\
\hline Gil 엉 el 20018 & 119.4 & 3.16 & 35 & 1134 & $2.4 ?$ & 35 & $72 \%$ & $6.0014 .67,7.33$ & & & - & \\
\hline Hossain at al 2010 & 114.9 & 128 & 40 & 100.1 & 15.9 & 40 & $4.0 \%$ & $14.80[8.47,21.13$ & & & & \\
\hline indeli et el 2018 & 123 & 5.2 & 50 & 120 & 8.7 & 50 & 6.48 & $3.00 \mu .19,5.81$ & & & & \\
\hline Kistiresthe et el 2020 & 1079 & 19.7 & 36 & 1181 & 143 & 37 & $32 \%$ & $-10.30+18.21,-2.39$ & & & & \\
\hline Lin et el 2020 & 99.6 & 13.46 & 103 & 98.16 & 13.9 & 271 & $6.2 \%$ & $0.44+2.84,3.02$ & & & & \\
\hline Papagiennia atol 2016 & 117.65 & 3.08 & 24 & 117.9 & 327 & 22 & 708 & $-0.05+180,1.79$ & & & & \\
\hline $\begin{array}{l}\text { Wang et el } 2021 \\
\text { Sutisotal ( } 95 \mathrm{~S} \mathrm{Cl})\end{array}$ & 12224 & 4.45 & $\begin{array}{l}126 \\
450\end{array}$ & 123.78 & 6.00 & $\begin{array}{l}126 \\
617\end{array}$ & $\begin{array}{r}7.2 \% \\
48.8 \%\end{array}$ & $\begin{array}{r}-1.54+2.80,-0.23 \\
1.92[-1.18,5.03)\end{array}$ & & & & \\
\hline \multicolumn{13}{|c|}{ 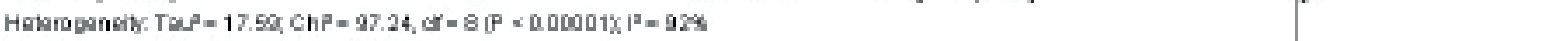 } \\
\hline \multicolumn{13}{|l|}{ 1.3.3 Poom miatern } \\
\hline Bese ef el 2018 & 123.7 & 14.8 & 150 & 127.1 & 16.1 & 150 & $6.9 \%$ & $-3.40+6.90,0.10$ & & & & \\
\hline batra ct al 2020 & 118 & B.6 & 53 & 116 & 93 & 59 & 6.08 & $200|1,41,5,41|$ & & & & \\
\hline Sriat el 2020 & 11372 & 8.43 & 280 & 11272 & 8.18 & 297 & $7.1 \%$ & $1.00+0.42,2.42$ & & & & \\
\hline Sutiletal (865 cD & & & 493 & & & 440 & 18.15 & 0.07 [-2.65, 2.79] & & & & \\
\hline \multicolumn{13}{|c|}{ 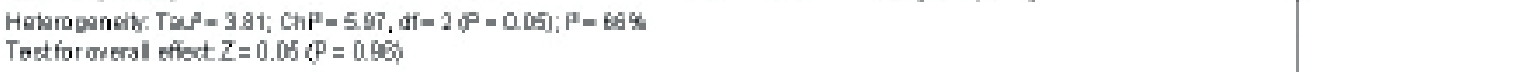 } \\
\hline Total (35s Ci) & & & 14ab & & & 1653 & $160.0 \mathrm{~s}$ & $2.02[4.13,3.10]$ & & & & \\
\hline \multicolumn{8}{|c|}{ 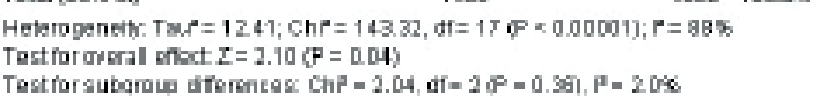 } & & -50 & Fawaurs P\& & Fawurs MP & 60 \\
\hline
\end{tabular}

Figure 4. - Forest plot of comparison of ROM after one year, two years and midterm follow up.

knees (387 PS; 440 MP). Heterogeneity analysis demonstrated moderate statistical evidence for variation within the study $\left(\mathrm{I}^{2}=75 \%\right)$. Data pooled by random-effects model reported a non significant difference in favour of the PS cohort. The cumulative $\mathrm{MD}$ was insignificant at -0.15 (95\% CI, 2.30-2.60; $\mathrm{P}$ $=0.66)($ Fig. 6).

Overall, four studies including 289 knees (151 PS; 138 MP) reported on FJS after one year. They reported a non significantly better FJS score for the MP cohort at one year. The heterogeneity analysis demonstrated moderate statistical evidence for variation within the study $\left(\mathrm{I}^{2}=68 \%\right)$. The cumulative MD was insignificant at 3.77 (95\% CI, -7.72-15.25; $\mathrm{P}=0.52$ ) (Fig. 7).

Three studies totalling 175 knees (88 PS; 87 MP) reported on postoperative FJS at two years post-op. They reported a significantly superior FJS with the
MP cohort versus the PS cohort. The heterogeneity analysis demonstrated high statistical evidence for variation within the study $\left(\mathrm{I}^{2}=86 \%\right)$. The cumulative MD was insignificant at 8.94 (95\% CI, -2.82-20.71; $\mathrm{P}<0.06$ ) (Fig. 7).

Three studies including 227 knees (112 PS; 115 MP) recorded WOMAC score after one year. They reported an improved but nonsignificant WOMAC score for the MP group at one year follow-up. The heterogeneity analysis demonstrated moderate statistical evidence for variation within the study $\left(\mathrm{I}^{2}\right.$ $=56 \%$ ). The cumulative $\mathrm{MD}$ was insignificant at 1.88 (95\% CI, 4.52-8.29: $\mathrm{P}=0.56$ ) (Fig. 8).

Two studies totalling 332 knees (166 PS; 166 MP) reported on WOMAC score after two years. The MP cohort recorded an insignificantly higher functional KSS compared with the PS group. The heterogeneity analysis demonstrated moderate 


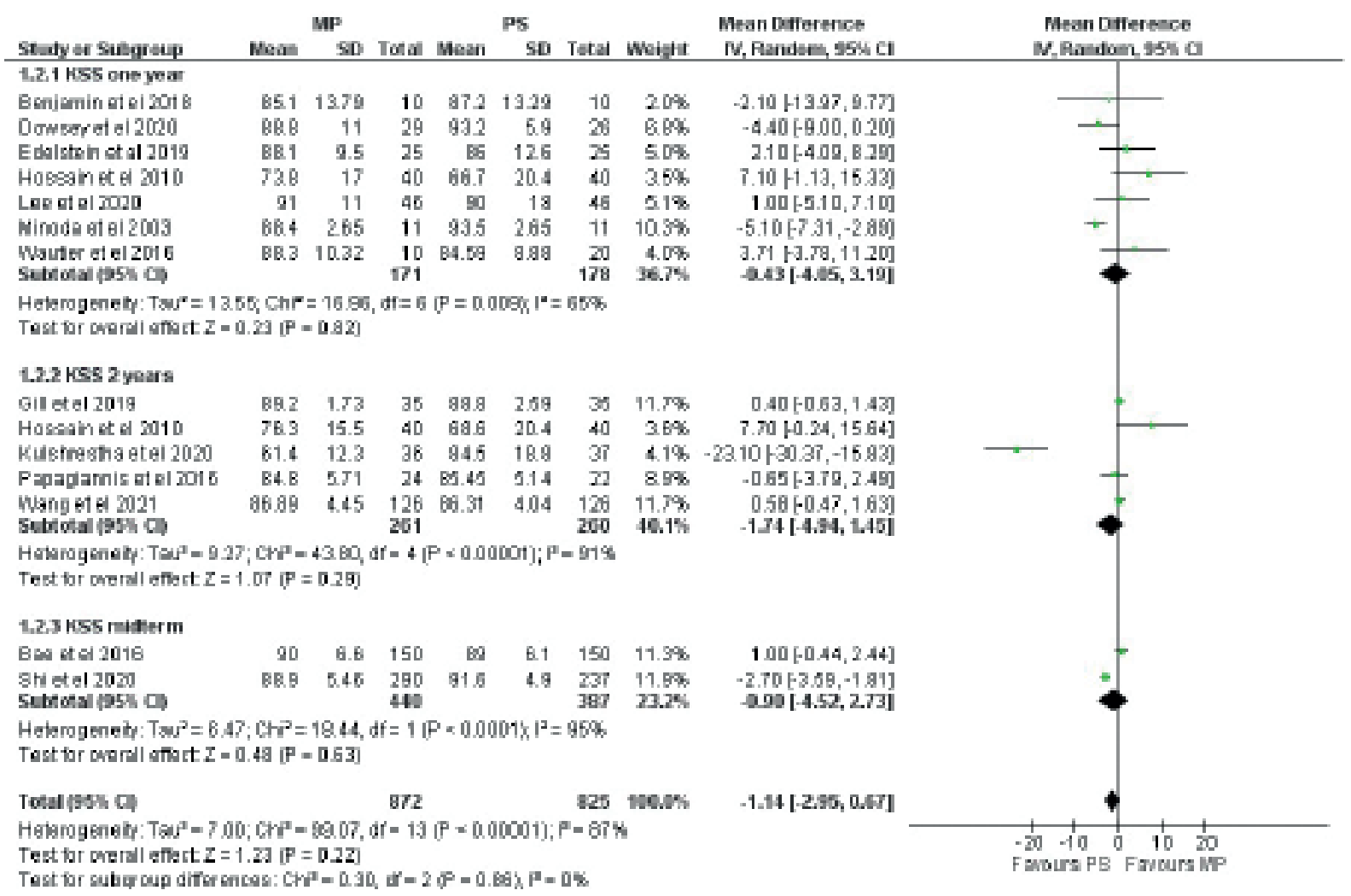

Figure 5. - Forest plot of comparison of KSS between MP and PS knee after one year, two years and midterm follow up.

statistical evidence for variation within the study $\left(\mathrm{I}^{2}\right.$ $=59 \%$ ). The cumulative MD was insignificant at 1.18 (95\% CI, -7.40-5.04; $\mathrm{P}=0.71$ ) (Fig. 8).

Two studies reported on midterm WOMAC scores encompassing a total of 827 knees (387 PS; $440 \mathrm{MP}$ ). Heterogeneity analysis demonstrated no statistical evidence for variation within the study $\left(\mathrm{I}^{2}=0 \%\right)$. Data pooled by random-effects model reported a significant higher score in favour of the MP cohort. The cumulative MD was insignificant at 1.77 (95\% CI, -2.70-0.85; $\mathrm{P}=0.0002$ ) (Fig. 8).

Overall, five studies including 308 knees (151 PS; 157 MP) reported on OKS after one year. They reported an insignificantly higher OKS for the PS cohort at one year follow-up. Heterogeneity analysis demonstrated low statistical evidence for variation within the study $\left(\mathrm{I}^{2}=16 \%\right)$. The cumulative MD was insignificant at $-0.43(95 \% \mathrm{CI},-2.01-1.16$; $\mathrm{P}=$ 0.60) (Fig. 9).

Four studies involving 336 knees (168 PS; 168 MP) recorded OKS at two years follow-up. They reported an insignificantly higher OKS within the MP cohort. The heterogeneity analysis demonstrated low statistical evidence for variation within the study $\left(\mathrm{I}^{2}=30 \%\right)$. The cumulative MD was insignificant at -0.41 (95\% CI, -0,22-1,05; $\mathrm{P}=0.20)$ (Fig. 9).

\section{DISCUSSION}

The most important finding in this review was that the post-operative ROM was insignificantly higher in the MP cohort compared with the PS cohort. The MP design is a combination of a congruent medial compartment and a less congruent lateral compartment. It is still somewhat controversial as to whether this design achieves normal physiological knee kinematics. The MP knee showed improved kinematics in vivo which might explain the better $\mathrm{KSS}$ at short and midterm follow up (30). However, after examining multiple different implant designs, some authors have claimed that around $50 \%$ of patients achieve medial pivoting gait and 
676 H. ElBARDESY, H.M. SALAMAH, A. MCLEOD, P.K. THADA, E.R. MOHAMMED, F.A. HANIFA, M. ROSHDY, S. GUERIN

\begin{tabular}{|c|c|c|c|c|c|c|c|c|c|}
\hline \multirow[b]{2}{*}{ Stuey or Siecios } & \multicolumn{3}{|c|}{ MP } & \multicolumn{3}{|c|}{ PS } & \multicolumn{2}{|c|}{ Mean Differesce } & \multirow{2}{*}{$\begin{array}{l}\text { Nean Deflerence } \\
\text { N. Rastom. } 95 \% \mathrm{CI}\end{array}$} \\
\hline & Mean & So & Total & Mean & $\$ 0$ & Tetal & Weinif & W. Raatom, 95\% C & \\
\hline \multicolumn{9}{|c|}{ 1.3. I Fusclional KSS one year } & \\
\hline Dowsey et el 2030 & 55.7 & 173 & 29 & 70.4 & 19.4 & 26 & $2.7 \%$ & $-4.70[14.45,5.06]$ & \\
\hline Edelsisin at 32018 & 81.4 & 179 & 26 & 77.4 & 22.6 & 26 & $2.1 \%$ & $4.00[-7.27,16.27]$ & \\
\hline Hosasin at al 2010 & 73.8 & 17 & 40 & $67 \mathrm{~A}$ & 23.5 & 40 & $3.1 \%$ & $600[-292,1499]$ & \\
\hline Lee et. ex 2020 & 6 & 21 & 46 & 60 & 22 & 46 & $3.2 \%$ & $-200[-10.79,6.79]$ & \\
\hline honods of el 2000 & 87.2 & 6.66 & 11 & 85.6 & 6.3 & 11 & $7.4 \%$ & $-8.20+13.47,-3.13]$ & \\
\hline Sutetal (ASH CD & & & 151 & & & 148 & t8.5. & $-178[-7.60,4.04]$ & \\
\hline \multicolumn{10}{|c|}{ 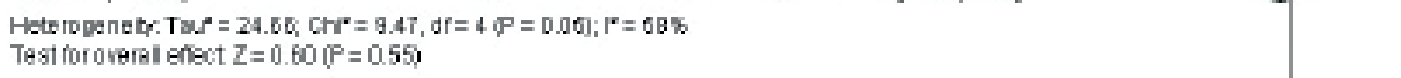 } \\
\hline \multicolumn{10}{|c|}{1,32 Fusctionul K5s 2 years } \\
\hline Hossin et ex 2010 & 71.4 & 15.8 & 40 & 69 & 24.6 & 40 & $3.0 \%$ & $3.40[-5.71,12.51]$ & \\
\hline Kulahrestia et el 2020 & 63.8 & 8.4 & 36 & 59.4 & 15.6 & 37 & 5.49 & $4.40[-133,10.13]$ & \\
\hline Papspiarnis ef el 2010 & 93.70 & 0.01 & 24 & 85.9 & 5.35 & 22 & $13.7 \%$ & $-2.15+5.15,0.80]$ & \\
\hline Whand eter 2021 & 80.54 & 6.21 & 126 & g7gs & 553 & 1260 & 20.34 & $0.611-0.84,2067$ & \\
\hline Sistetal (95) CD & & & 2265 & & & 225 & $434 / \mathrm{h}$ & $0.42[-1.90,2.75 \mid$ & \\
\hline \multicolumn{10}{|c|}{ 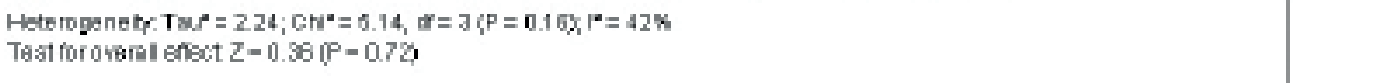 } \\
\hline \multicolumn{10}{|c|}{ 1.3.3 Functional KSS midierm } \\
\hline 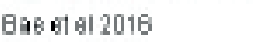 & 85.6 & 0.5 & 150 & $8 ?$ & 6.8 & 150 & $18.0 \%$ & $-1.40+3.15,0.35]$ & $\Rightarrow$ \\
\hline Shi of al 2020 & 79.1 & 10.35 & 200 & $\mathrm{rg}$ & 9.8 & 737 & $19.1 \%$ & $1.10+0.63 .283]$ & \\
\hline Satetal $655 \mathrm{co}$ & & & 460 & & & 387 & 38.15 & $-0.16(-2.60,2.36)$ & \\
\hline \multirow{2}{*}{\multicolumn{10}{|c|}{ 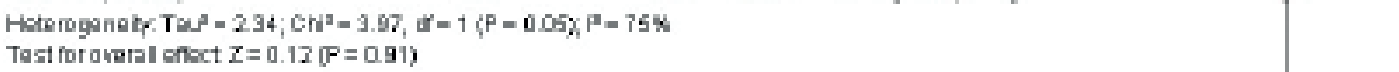 }} \\
\hline & & & & & & & & & \\
\hline \multirow{2}{*}{\multicolumn{7}{|c|}{ 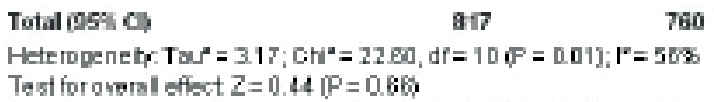 }} & $1000 \mathrm{~m}$ & $-0.381 .208,1.321$ & \\
\hline & & & & & & & & & 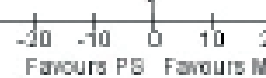 \\
\hline
\end{tabular}

Figure 6. - Forest plot of comparison of functional KSS between MP and PS knee after one year, two years and midterm follow up.

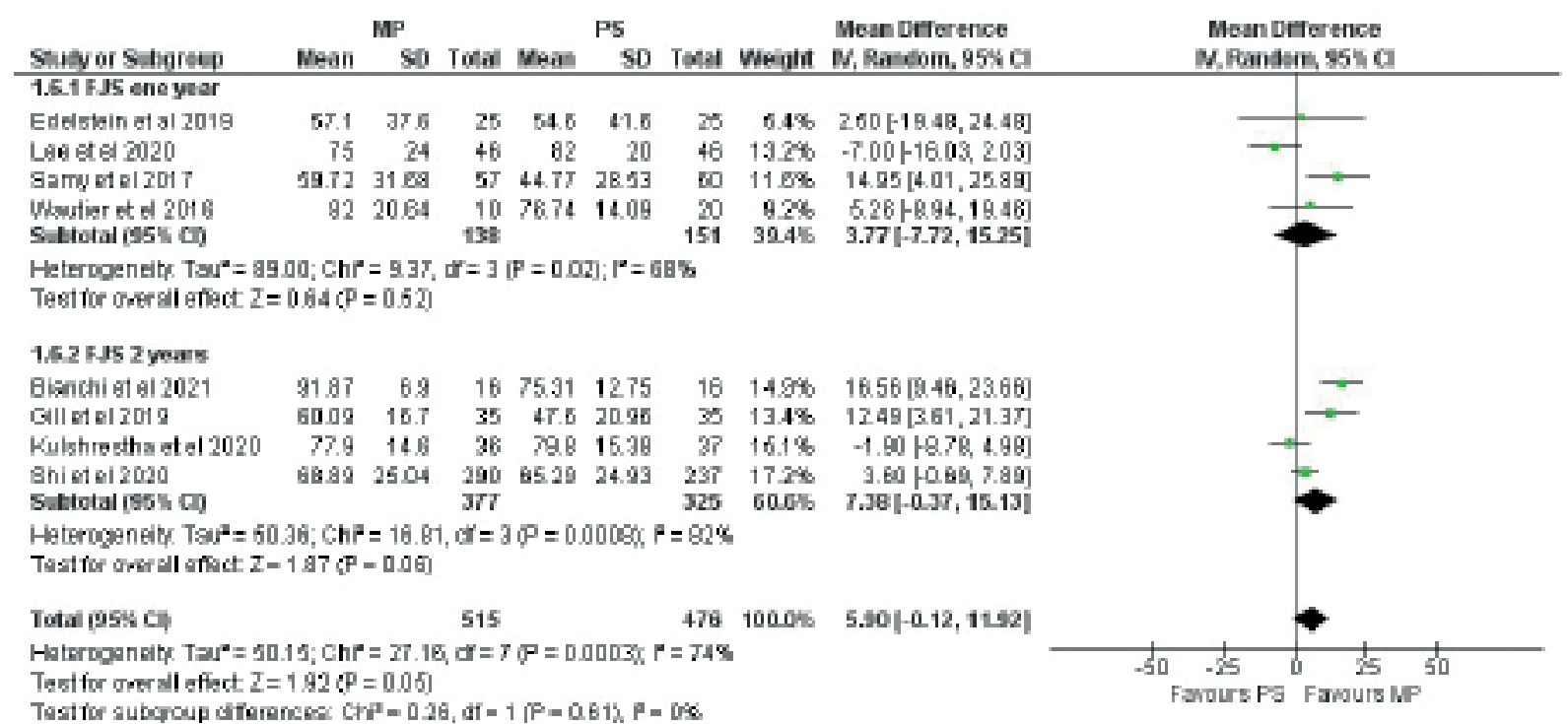

Figure 7. - Forest plot of comparison of FJS between the MP and the PS knee after one year and two years follow-up.

satisfactory knee flexion regardless of the knee prothesis they received, suggesting that knee motion doesn't absolutely correlate with the prothesis design (42). Others authors couldn't report any significant kinematic or clinical advantage to the MP knee when compared to other implants at long term $(43,44)$. Previous studies that compared the MP and PS protheses were controversial. Some authors 


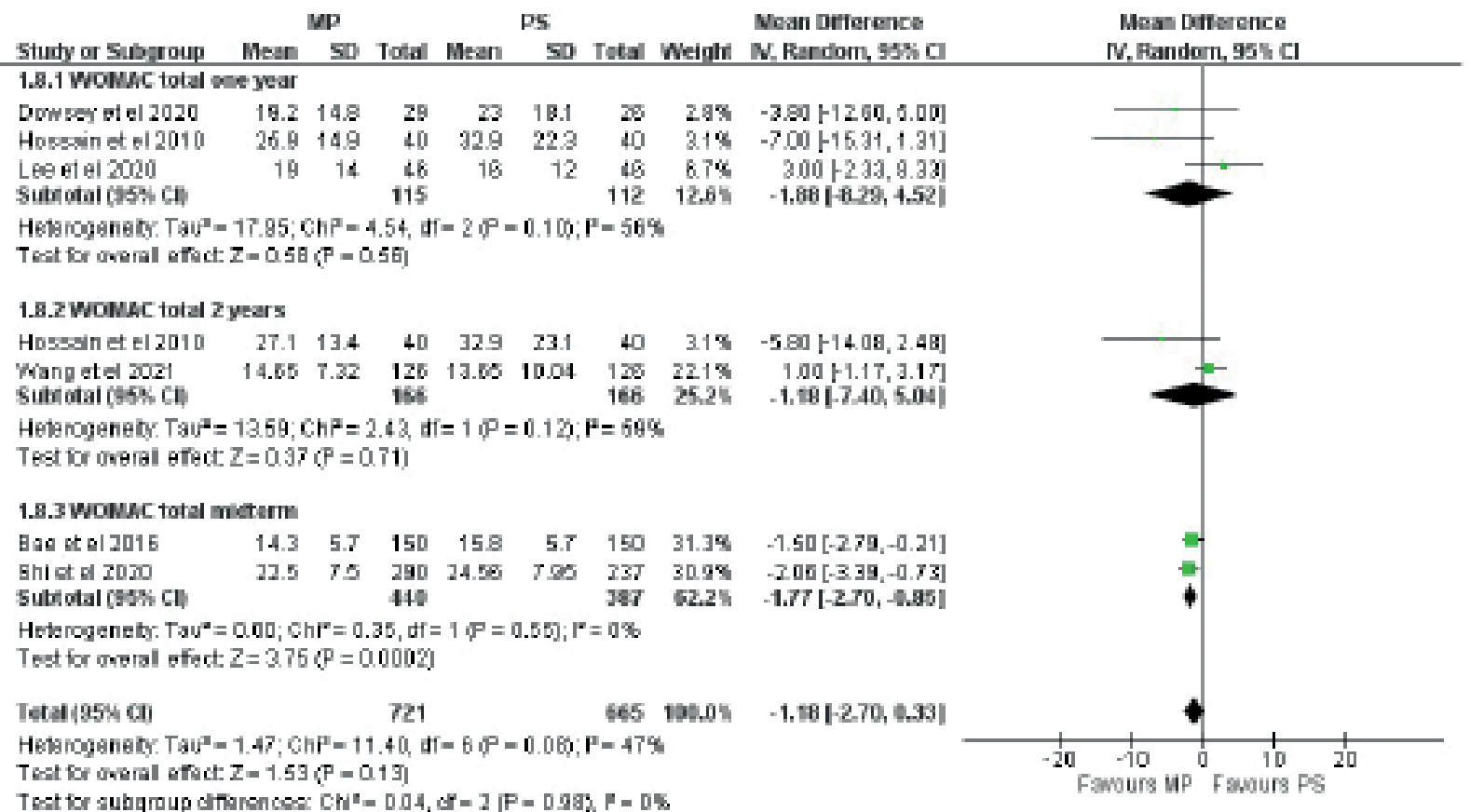

Figure 8. - Forest plot of comparison of WOMAC scores between the MP and the PS knee at one year, two years and midterm follow-up.

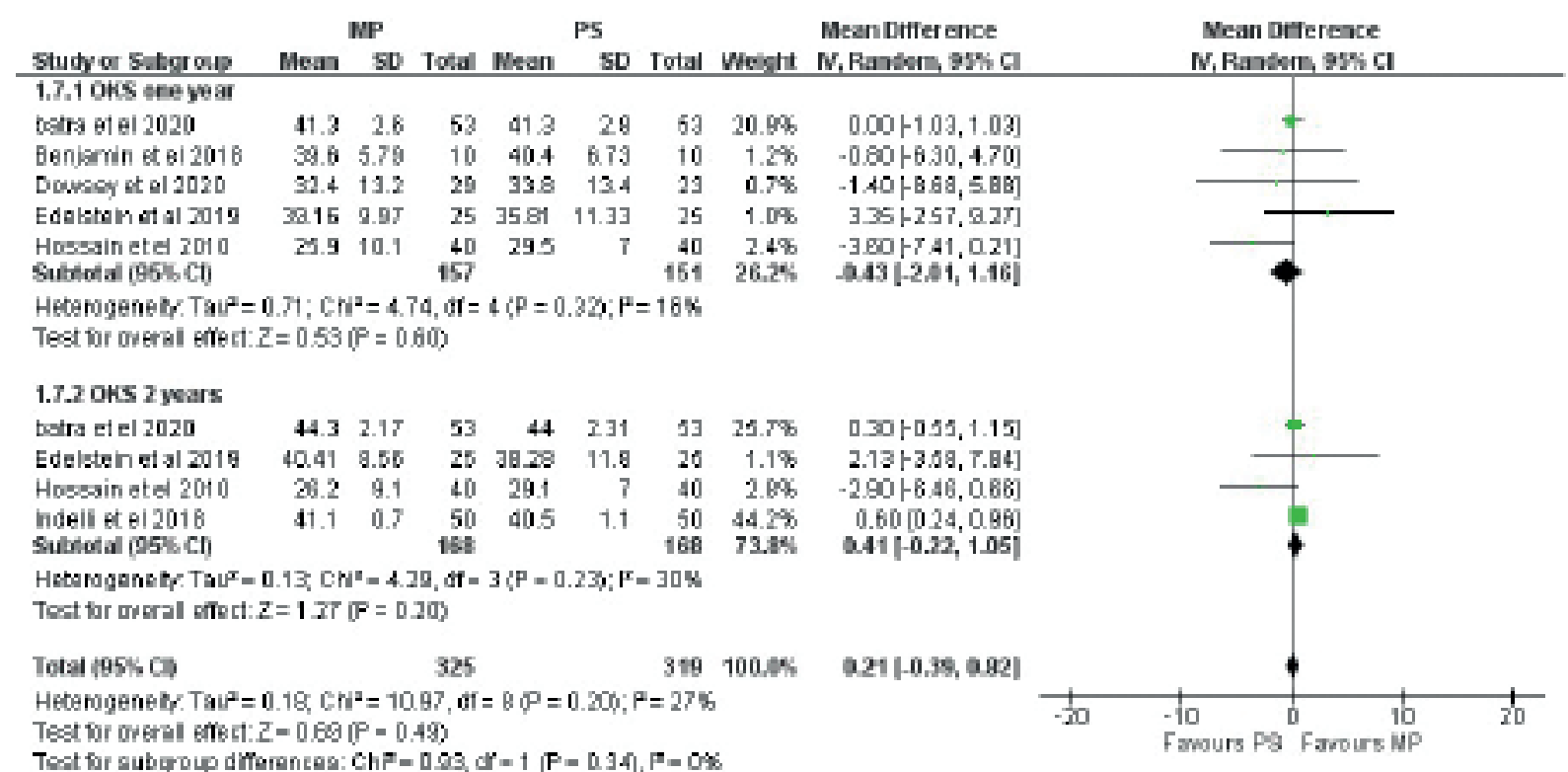

Figure 9. - Forest plot of comparison of OKS between the MP and the PS knee at one year and two years follow-up.

couldn't find any significant difference between the MP and PS knee in the context of patient satisfaction at one year follow-up (23). However, other studies reported better FJS in the patients with the MP knee which did not correlate with our results in this review (21,31). Dowsey et al. (27), reported significatly superior pain, function, and quality of life outcomes among the MP cohort in their Randomised control 
trial (RCT). In contrast, Kim et al.. (45) reported worse outcomes with the MP knee after two years follow. However, this study received serious criticism due to the infection rate associated with the MP cohort and the study's methodology in general $(46,47)$. Batra et al.. (30), examined the kinematics of the MP and PS knee and demonstrated that the MP prothesis showed significantly better kinematics than the PS knee but reported no significant difference in the ROM between the two groups. Some researchers that assessed the kinematics of the prosthetic knees by using intraoperative sensors and CT guided navigation systems reported a higher satisfaction rate with the MP design $(48,49)$. The Australian Orthopaedic Association National Joint Replacement Registry (AOANJRR) reported encouraging outcomes for the MP TKA, with the same revision rate as the PS knee (1). Edelstein et al.. (26), reported mid flexion stability in the sagittal plane with the MP knee in comparison with a PS construct. Moreover, they reported improved patient function during standing in flexion activities in the MP cohort. To our knowledge, there is only one meta-analysis (17) that compared the pivot and the non-pivot knee designs. They analysed only the KSS and WOMAC score due to the limited number of studies included and they were unable to reach any significant conclusion in their review. To our knowledge this is the first study to use meta-analysis and systematic review to investigate the ROM and PROMs between the MP and PS knees.

One of the strengths of our study is the large number of patients included in our analysis (3837 knees). As well as this a significant portion or our included studies are modern, with nineteen published between 2018 and 2021. In terms of study limitations, the data used in this study was obtained from several studies reporting the ROM and PROMs between the MP and PS knees. The techniques and materials used in these studies were similar but not identical. Another limitation is the inclusion of eight retrospective studies in the metaanalysis. The observational patterns associated with retrospective cohort studies are more susceptible to bias in data collection, and are confronted by the incompetence to control for all the variables measured between the different cohorts included in each study. Another source of limitation was the lack of long-term follow-up. While the studies included reported scores for up to 8 years followup, there is a paucity of data beyond this. We would recommend more RCTs with a long term follow up period examining these two prosthetic designs.

\section{CONCLUSION}

In terms of ROM, KSS, OKS and FJS this meta-analysis suggests insignificant better shortterm results for the MP knee compared with the PS prothesis. The MP implant also showed a significantly superior WOMAC score at short-term follow-up. An extended follow-up period is required to evaluate whether the MP knee is superior than the PS in the long-term.

\section{REFERENCES}

1. Australian Orthopaedic Association National Joint Replacement Registry. Australian Orthopaedic Association National Joint Replacement Registry (AOANJRR). Hip, knee \& shoulder arthroplasty - Annual Report 2018. 2018; 1-444. Available from: www.aoa.org.au

2. Bourne RB, Chesworth BM, Davis AM, Mahomed NN, Charron KDJ. Patient Satisfaction after Total Knee Arthroplasty: Who is Satisfied and Who is Not? Clin Orthop Relat Res [Internet]. 2010; 468(1): 57-63.

3. Beach A, Regazzola G, Neri T, Verheul R, Parker D. The effect of knee prosthesis design on tibiofemoral biomechanics during extension tasks following total knee arthroplasty. Knee [Internet]. 2019; 26(5): 1010-9.

4. van Duren BHH, Pandit H, Beard DJJ, Zavatsky ABB, Gallagher JAA, Thomas NPP, et al. How effective are added constraints in improving TKR kinematics? $J$ Biomech [Internet]. 2007; 40(SUPPL. 1): S31-7.

5. Elbardesy H, McLeod A, Gul R, Harty J. Midterm results of modern patellofemoral arthroplasty versus total knee arthroplasty for isolated patellofemoral arthritis: systematic review and meta-analysis of comparative studies. Arch Orthop Trauma Surg [Internet]. 2021.

6. Dorr LD, Ochsner JL, Gronley J, Perry J. Functional comparison of posterior cruciate-retained versus cruciatesacrificed total knee arthroplasty. Clin Orthop Relat Res [Internet]. 1988; (236): 36-43.

7. Broberg JS, Ndoja S, MacDonald SJ, Lanting BA, Teeter MG. Comparison of Contact Kinematics in PosteriorStabilized and Cruciate-Retaining Total Knee Arthroplasty at Long-Term Follow-Up. J Arthroplasty [Internet]. 2020; 35(1): 272-7. 
8. Wolterbeek N, Nelissen RGHH, Valstar ER. No differences in in vivo kinematics between six different types of knee prostheses. Knee Surgery, Sport Traumatol Arthrosc [Internet]. 2012; 15; 20(3): 559-64.

9. Oshima Y, Iizawa N, Takai S, Majina T. The Optimal Distraction Force to Evaluate Tibiofemoral Joint Gaps in Posterior Stabilized-Total Knee Arthroplasty. J Nippon Med Sch [Internet]. 2021; doi: 10.1272/jnms.JNMS.2021_88519.

10. Bae JH, Lee JW, Kim SH, Kim SG, Jeon YS, Choi JS. Femoral matched tibia component rotation has little effect on the tibial torsion after total knee arthroplasty. Knee Surgery, Sport Traumatol Arthrosc [Internet]. 2021 Jan 15.

11. Dubin JA, Muskat A, Westrich GH. Design Modifications of the Posterior-Stabilized Knee System May Reduce Anterior Knee Pain and Complications Following Total Knee Replacement. HSS $J{ }^{\circledR}$ [Internet]. 2020; 16(S2): 3448 .

12. Zingde SM, Leszko F, Sharma A, Mahfouz MR, Komistek RD, Dennis DA. In Vivo Determination of Cam-Post Engagement in Fixed and Mobile-bearing TKA. Clin Orthop Relat Res [Internet]. 2014; 472(1): 254-62.

13. Wang $Z$, Zhang $Y$ qing, Ding $C$ rong, Wang $Y$ zhen, Xu H. Early Patellofemoral Function of Medial Pivot Prostheses Compared with Posterior-Stabilized Prostheses for Unilateral Total Knee Arthroplasty. Orthop Surg. 2021; (February 2020): 1-9.

14. Gray HA, Guan S, Young TJ, Dowsey MM, Choong PF, Pandy MG. Comparison of posterior-stabilized, cruciateretaining, and medial-stabilized knee implant motion during gait. J Orthop Res. 2020; 38(8): 1753-68.

15. Tanifuji O, Sato T, Kobayashi K, Mochizuki T, Koga Y, Yamagiwa $\mathbf{H}$, et al. Three-dimensional in vivo motion analysis of normal knees employing transepicondylar axis as an evaluation parameter. Knee Surgery, Sport Traumatol Arthrosc [Internet]. 2013 ; 21(10): 2301-8.

16. Bianchi N, Facchini A, Mondanelli N, Sacchetti F, Ghezzi R, Gesi M, et al. Medial pivot vs posterior stabilized total knee arthroplasty designs: a gait analysis study. Med Glas. 2021; 18(1).

17. Young T, Dowsey MM, Pandy M, Choong PF. A Systematic Review of Clinical Functional Outcomes After Medial StabilizedVersus Non-Medial Stabilized Total Knee Joint Replacement. Front Surg. 2018; 5: 11; 5: 25.

18. Julian P.T. Higgins JT, Jacqueline Chandler, Miranda Cumpston TL, Welch MJP and VA. Cochrane Handbook for Systematic Reviews of Interventions. 2019. 241-284 p.

19. Wells GA, Shea B, O'Connell D, Peterson J, Welch V LM. The NewcastleeOttawa Scale (NOS) for Assessing the Quality of Non-randomized Studies in Meta-analysis. Ottawa Heal Res Inst. 2003; 1(1): 1-12.

20. Kulshrestha V, Sood M, Kanade S, Kumar S, Datta B, Mittal G. Early outcomes of medial pivot total knee arthroplasty compared to posterior-stabilized design: A randomized controlled trial. CiOS Clin Orthop Surg. 2020; 12(2): $178-86$.
21. Pritchett JW. Patients Prefer A Bicruciate-Retaining or the Medial Pivot Total Knee Prosthesis. J Arthroplasty [Internet]. 2011; 26(2): 224-8.

22. Pritchett JW. A comparison of the noise generated from different types of knee prostheses. J Knee Surg. 2013; 26(2): 101-4.

23. Lee QJ, Wai Yee EC, Wong YC. No difference in patient preference for medial pivot versus posterior-stabilized design in staged bilateral total knee arthroplasty: a prospective study. Knee Surgery, Sport Traumatol Arthrosc [Internet]. 2020; 28(12): 3805-9.

24. Hossain F, Patel S, Rhee SJ, Haddad FS. Knee arthroplasty with a medially conforming ball-and-socket tibiofemoral articulation provides better function. Clin Orthop Relat Res. 2011; 469(1): 55-63.

25. Gill UN, Shiraz HM, Rehman MKU, Malik AL, Mian MH. Comparison of functional outcome of medial pivot total knee arthroplasty with posterior stabilizing (PS) total knee arthroplasty - A randomized trial. Pakistan J Med Heal Sci. 2019; 13(2): 385-8.

26. Edelstein AI, Bhatt S, Wright-Chisem J, Sullivan R, Beal M, Manning DW. The Effect of Implant Design on Sagittal Plane Stability: A Randomized Trial of Medialversus Posterior-Stabilized Total Knee Arthroplasty. J Knee Surg. 2020; 33(5): 452-8.

27. Dowsey MM, Gould DJ, Spelman T, Pandy MG, Choong PF. A Randomized Controlled Trial Comparing a Medial Stabilized Total Knee Prosthesis to a Cruciate Retaining and Posterior Stabilized Design: A Report of the Clinical and Functional Outcomes Following Total Knee Replacement. J Arthroplasty. 2020; 35(6): 1583-1590.e2.

28. Benjamin B, Pietrzak JRT, Tahmassebi J, Haddad FS. A functional comparison of medial pivot and condylar knee designs based on patient outcomes and parameters of gait. Bone Jt J. 2018; 100B(1): 76-82.

29. Yuan D, Zhang QS, Zhang K. et al. Total Knee Arthroplasty Using a Medial Pivot or Posterior CruciateStabilizing Prosthesis in Chinese Patients. J Knee Surg. 2020; 33(9): 892-8.

30. Batra S, Malhotra R, Kumar V, Srivastava DN, Backstein D, Pandit H. Superior patient satisfaction in medial pivot as compared to posterior stabilized total knee arthroplasty: a prospective randomized study. Knee Surgery, Sport Traumatol Arthrosc [Internet]. 2020; (0123456789): doi: 10.1007/s00167-020-06343-4.

31. Samy DA, Wolfstadt JI, Vaidee I, Backstein DJ. A Retrospective Comparison of a Medial Pivot and PosteriorStabilized Total Knee Arthroplasty With Respect to PatientReported and Radiographic Outcomes. J Arthroplasty. 2018; 33(5): 1379-83.

32. Lin Y, Chen X, Li L, Li Z, Zhang Y, Fan P. Comparison of Patient Satisfaction Between Medial Pivot Prostheses and Posterior-Stabilized Prostheses in Total Knee Arthroplasty. Orthop Surg. 2020; 12(3): 836-42.

33. Papagiannis GI, Roumpelakis IM, Triantafyllou AI, Makris IN, Babis GC. No Differences Identified 
in Transverse Plane Biomechanics Between Medial Pivot and Rotating Platform Total Knee Implant Designs. $J$ Arthroplasty [Internet]. 2016; 31(8): 1814-20.

34. Zhang $Z$ an, Feng $H$, Yan $W$ ning, Li $H$ yan, Zhang $H$ ning, Bai $\mathbf{H}$ jun, et al. Comparison of Postoperative Effects between Medial Pivot Prosthesis and Posterior Stabilized Prosthesis. Orthop Surg. 2020; 12(6): 1843-53.

35. Shakespeare D, Ledger M, Kinzel V. Flexion after total knee replacement. A comparison between the Medial Pivot knee and a posterior stabilised implant. Knee. 2006; 13(5): 371-3.

36. Noble PC, Scuderi GR, Brekke AC, Sikorskii A, Benjamin JB, Lonner JH., et al. Development of a New Knee Society Scoring System. Clin Orthop Relat Res [Internet]. 2012; 470(1): 20-32.

37. Gill JR, Corbett JA, Wastnedge E, Nicolai P. Forgotten Joint Score: Comparison between total and unicondylar knee arthroplasty. Knee [Internet]. 2021; 29: 26-32.

38. Bellamy N, Buchanan WW, Goldsmith $\mathrm{CH}$, Campbell J, Stitt LW. Validation study of WOMAC: a health status instrument for measuring clinically important patient relevant outcomes to antirheumatic drug therapy in patients with osteoarthritis of the hip or knee. J Rheumatol [Internet]. 1988; 15(12): 1833-40.

39. Ko Y, Lo N-N, Yeo S-J, Yang K-Y, Yeo W, Chong H-C, et al. Comparison of the responsiveness of the SF-36, the Oxford Knee Score, and the Knee Society Clinical Rating System in patients undergoing total knee replacement. Qual Life Res [Internet]. 2013; 22(9): 2455-9.

40. (2014) RM (RevMan) [Computer P, Centre V 5. 3. CTNC, Collaboration. TC. Review Manager V.5.0.

41. Piuzzi NS. Patient-reported outcomes at 1 and 2 years after total hip and knee arthroplasty: what is the minimum required follow-up? Arch Orthop Trauma Surg [Internet]. 2021 [cited 2021 Mar 15].
42. Dennis DA, Komistek RD, Mahfouz MR, Haas BD, Stiehl JB. Conventry Award Paper: Multicenter Determination of In Vivo Kinematics After Total Knee Arthroplasty. Clin Orthop Relat Res [Internet]. 2003; 416: 37-57.

43. Bordini B, Ancarani C, Fitch DA. Long-term survivorship of a medial-pivot total knee system compared with other cemented designs in an arthroplasty registry. J Orthop Surg Res [Internet]. 2016; 11(1): 44.

44. Karachalios T, Varitimidis S, Bargiotas K, Hantes M, Roidis N, Malizos KN. An 11- to 15-year clinical outcome study of the Advance Medial Pivot total knee arthroplasty. Bone Joint J [Internet]. 2016; 98-B(8): 1050-5.

45. Kim Y-H, Yoon S-H, Kim J-S. Early Outcome of TKA with a Medial Pivot Fixed-bearing Prosthesis is Worse than with a PFC Mobile-bearing Prosthesis. Clin Orthop Relat Res [Internet]. 2009; 467(2): 493-503.

46. Scott G. Letter to the Editor: Early Outcome of TKA with a Medial Pivot Fixed-bearing Prosthesis is Worse than with a PFC Mobile-bearing Prosthesis. Clin Orthop Relat Res [Internet]. 2009; 467(3): 855-6.

47. Pritchett JW. Letter to the Editor: Early Outcome of TKA with a Medial Pivot Fixed-bearing Prosthesis Is Worse Than With a PFC Mobile-bearing Prosthesis. Clin Orthop Relat Res [Internet]. 2009; 467(1): 303-303.

48. Nishio Y, Onodera T, Kasahara Y, Takahashi D, Iwasaki N, Majima T. Intraoperative Medial Pivot Affects Deep Knee Flexion Angle and Patient-Reported Outcomes After Total Knee Arthroplasty. J Arthroplasty [Internet]. 2014; 29(4): 702-6.

49. Warth LC, Ishmael MK, Deckard ER, Ziemba-Davis M, Meneghini RM. Do Medial Pivot Kinematics Correlate With Patient-Reported Outcomes After Total Knee Arthroplasty? J Arthroplasty [Internet]. 2017; 32(8): 24116. 